\title{
Adherence to Low-Carbohydrate Diets in Patients with Diabetes: A Narrative Review
}

\author{
Nitya Kalyani Kumar $\mathbb{D}^{\prime}$, Jennifer D Merrill', Scott Carlson', Jashalynn German', \\ William S Yancy Jr $\mathbb{D}^{3}$ \\ 'Division of Endocrinology, Diabetes, \& Metabolism, Duke University, Durham, NC, USA; ${ }^{2}$ Division of Endocrinology, Diabetes, \& Metabolism, Ohio \\ State University, Columbus, OH, USA; ${ }^{3}$ Duke Lifestyle and Weight Management Center and Division of General Medicine, Duke University, Durham, \\ NC, USA
}

Correspondence: Nitya Kalyani Kumar, 30 Duke Medicine Circle Clinic IA, Durham, NC, 277I0, USA, Email nitya.kumar@duke.edu

\begin{abstract}
Evidence suggests that low carbohydrate ( $<130 \mathrm{~g}$ /day of carbohydrate) (LCD) and very low carbohydrate, ketogenic diets (typically $<50 \mathrm{~g} /$ day of carbohydrate) (VLCKD) can be effective tools for managing diabetes given their beneficial effects on weight loss and glycemic control. VLCKD also result in favorable lipid profile changes. However, these beneficial effects can be limited by poor dietary adherence. Cultural, religious, and economic barriers pose unique challenges to achieving nutritional compliance with LCD and VLCKD. We review the various methods for assessing adherence in clinical studies and obstacles posed, as well as potential solutions to these challenges.
\end{abstract}

Keywords: low carbohydrate diet, ketogenic diet, adherence, type 2 diabetes

\section{Introduction}

The number of people with type 2 diabetes has nearly quadrupled from 1980 to 2014 with the prevalence now estimated to be $8.5 \%$ of the global population. ${ }^{1}$ Diabetes is associated with increased morbidity and mortality, but the development and progression of weight-related type 2 diabetes can be delayed with exercise, diet, and weight loss of $5-10 \%{ }^{2-5}$ Guidelines from the American Diabetes Association and Academy of Nutrition and Dietetics therefore recommend that overweight adults with type 2 diabetes reduce energy intake to promote weight loss. ${ }^{6-9}$ However, the optimal dietary approach to weight loss is widely debated, among both health professionals and the lay public. A preponderance of data suggests that low carbohydrate diets (LCD) and very low carbohydrate, ketogenic diets (VLCKD) can be effective tools for weight loss, A1c reduction, blood pressure lowering, and improving triglycerides and HDL-C in trial settings. ${ }^{7,10}$ An emerging body of evidence, however, suggests that better adherence to a weight-reducing diet, rather than the diet composition itself is an important factor for weight loss success. ${ }^{11,12}$

Dietary predictors of adherence vary based on the type of diet. In one study examining both qualitative and quantitative methods among adults following a restrictive dietary pattern, adherence varied significantly between dietary groups, with those following a vegan and vegetarian-based diet demonstrating particularly high adherence and glutenfree and weight loss dieters being comparably low. Four consistent predictors of adherence were noted. Self-efficacy and social identification with one's dietary group positively predicting adherence, whereas mood or weight control as motivation for dietary choice had negative effects. ${ }^{13}$ Personality characteristics have also been linked to dietary adherence, with conscientiousness, lower levels of emotional eating, and openness to experience being associated with better adherence in weight-loss intervention programs. ${ }^{13-15}$ Mental health is known to play an important role as well. For those with celiac disease, depression and anxiety were associated with poor adherence to a gluten-free diet. ${ }^{13}$

There is no consensus on the amount of carbohydrates permitted for a LCD or VLCKD. Whether carbohydrates should include total carbohydrates versus net carbohydrates also remains debatable Net carbohydrates are calculated as total carbohydrates after excluding fiber and sugar alcohols (if applicable). For this review, studies were eligible for 
inclusion if they were randomized control trials including adults diagnosed with Type 2 diabetes, had a minimum intervention duration of 24 weeks, and if the intervention restricted the proportion or quantity of dietary carbohydrate to at least less than 50 grams of carbohydrate per day. Studies using active control diets were included. All forms of comparison diet that did not include carbohydrate restriction were permitted, including low-fat, high-carbohydrate, lowglycemic index, high-protein, Mediterranean and "healthy eating". Included studies also needed to report actual (selfreported or measured) carbohydrate intake during or at the end of the intervention. All countries were eligible but language was restricted to English. Studies that did not meet these criteria were excluded. Included studies did not differentiate between total versus net carbohydrates. This article seeks to review the literature related to adherence to LCD and VLCKD in people with type 2 diabetes based on the aforementioned criteria.

\section{A Brief History of Low Carbohydrate and Very Low Carbohydrate Diets}

The development of the VLCKD as a treatment for diabetes mirrors its use as a treatment for epilepsy. VLCKD was the standard of care for both diabetes and epilepsy prior to the development antidiabetic and antiepileptic drugs, respectively ${ }^{16-18}$ After the development of pharmacologic treatments, interest in dietary modification waned. However, medications failed to yield a universal cure, prompting a resurgence in research and clinical interest in LCD and VLCKD for the management of diabetes and epilepsy.

In 1921, the Mayo Clinic treated a series of epilepsy patients with a ketone-producing diet. This "ketogenic diet" was an effective alternative to fasting. ${ }^{18}$ First reported in 1925, the macronutrient distribution of the ketogenic diet for pediatric epilepsy consisted of $1 \mathrm{~g}$ protein per kilogram of body weight, 10-15 g of carbohydrates per day, and the remainder of the calories from fat. ${ }^{19}$ In these earliest reports of the diet in the treatment of epilepsy, dietary adherence played a significant role in seizure control. ${ }^{19}$

In the 1910s, Drs. Frederick Madison Allen and Elliot Proctor Joslin studied low carbohydrate, high-fat diets for the treatment of diabetes mellitus. ${ }^{17}$ Decreased urinary glucose output was a marker of efficacy. These diets consisted roughly of $70 \%$ fat and $10 \%$ carbohydrates. ${ }^{17}$ Prior to 1920 , poor dietary adherence in patients with diabetes was noted to result in recurrent admission and poor outcomes. ${ }^{20}$

The Atkins' diet, popularized in 1972, was the first ketogenic diet to gain widespread appeal for obesity management. Variants of the classical ketogenic diet (CKD) were developed to improve tolerability. These include the modified Atkins' $\operatorname{diet}(\mathrm{MAD})^{21,22}$ the medium-chain triglyceride diet, and the low glycemic index treatment. ${ }^{23}$ The MAD does not require an initial fasting period and is meant to be easy to follow. Fats are encouraged and carbohydrates limited to less than 20 grams per day. ${ }^{22,24}$ A meta-analysis evaluated the 15 studies of ketogenic diets for epilepsy in adults. ${ }^{23}$ The studies had a combined dropout rate of $45 \%$, with fewer patients completing the trials of the CKD (38\%) and better completion rates with the MAD $(56 \%) .{ }^{23}$ Of the patients who dropped out of the classical ketogenic diet studies, about $60 \%$ did so due to inability to adhere which was attributed to psychosocial reasons, fatigue and weight loss. ${ }^{25-28}$ Among the participants who failed to complete the MAD studies, about half discontinued the diet due to inability to adhere. When ketogenic diets are used to control epilepsy, adherence appears to be inversely related to the degree of dietary restriction. Side effects and psychosocial stressors also appear to impact dietary adherence in this setting.

\section{Defining Low Carbohydrate and Very Low Carbohydrate Diets and Their Clinical Benefits}

Dietary approaches for weight loss typically emphasize either decreasing carbohydrate intake, decreasing fat intake or decreasing calories/portions. ${ }^{29}$ A typical American diet consists of 2200 calories per day, with roughly $36-40 \%$ from fat, 16-18\% from protein, and $45 \%$ from carbohydrates. ${ }^{30-32}$ Traditional dietary guidelines for people with diabetes recommended that individuals consume $45-65 \%$ of daily energy intake from carbohydrates high in fiber. ${ }^{33}$ Randomized control trials generally define low carbohydrate diets as $<40-45 \%$ of calories from carbohydrate. ${ }^{7}$ However, more recently, studies have examined carbohydrate restricted eating patterns and found them to be safe. Some studies have also demonstrated metabolic benefits with LCDs and VLCKDs including weight loss, ${ }^{34,35}$ improved lipid profiles, ${ }^{7,36}$ better glycemic control, ${ }^{36}$ and reduced medication dependence in persons with diabetes. ${ }^{34,37}$ 
Although there are no accepted definitions for LCD or VLCKD, a selection of these eating patterns is reviewed herein based on the aforementioned criteria.

\section{Low Carbohydrate Diets (LCDs)}

These diets typically limit carbohydrate intake to $26-45 \%$ of daily caloric intake but do not seek to promote ketosis. ${ }^{7,38}$ These eating plans emphasize vegetables low in carbohydrate, fat from plant and animal foods, and protein from meat, poultry, fish, shellfish, eggs, cheese, plant-based sources like tofu and tempeh, and nuts. They avoid starchy and sugary foods such as pasta, rice, potatoes, bread, couscous, barley, polenta, cereal, crackers, and sweets. Popular LCDs include the South Beach and the Zone Diets. ${ }^{39,40}$ A meta-analysis of 9 studies showed significant weight loss in patients with type 2 diabetes on LCDs (less than 130g/day of carbohydrate) compared to control groups over long durations, and greater weight loss than control diets (typically low fat diets) at 6 months but not statistically greater at 12 months, possibly due to declining adherence over time. ${ }^{41,42}$ LCDs are associated with an increase in serum HDL-cholesterol and a decrease in fasting triglycerides when compared to low fat eating patterns. ${ }^{7,36}$ In an analysis of nine studies of dietary patterns each using a $<130 \mathrm{~g} /$ day from three months to two years, triglycerides were reduced and HDL was increased. LDL and total cholesterol changes were not statistically significant. ${ }^{41}$ Kirkpatrick analyzed eight meta-analyses of studies involving LCD and VLKCD in overweight or obese patients with prediabetes or type 2 diabetes and found a reduction in triglyceride levels, but no consistent changes in HDL, LDL, or total cholesterol though this was thought to be likely due to low adherence in all but one of the studies included. ${ }^{43}$ Fechner's meta-analysis compared the effects of varying degrees of carbohydrate reduction in LCDs on metabolic markers. Though only four of 37 included trials involved those with type 2 diabetes, the authors found that the degree of carbohydrate restriction was proportional to triglyceride reduction. ${ }^{44}$

In meta-analyses, there is a significant A1c reduction in people adhering to LCD compared to a high carb (fat restricted) diet with 2 years of follow-up. ${ }^{36,45}$ However, the A1c reduction appears to be due to the studies with carbohydrate restriction to less than $26 \%$ of energy, so this may not be generalizable. ${ }^{45}$ LCDs are associated with greater reductions in the number and dose of diabetes medications compared to other diets. ${ }^{45,46}$

\section{Very Low Carbohydrate Ketogenic Diets (VLCKDs)}

Ketogenic diets adapted for weight management and general wellness differ from those used to treat epilepsy. Although there is no standardized definition, these eating patterns are typically characterized by reducing dietary carbohydrates to $20-50$ g per day but sometimes are defined for study purposes as $<26 \%$ of daily calories. ${ }^{7,47}$ This goal is based on the level of carbohydrate reduction required to produce ketosis, which is typically $<50 \mathrm{~g} /$ day. ${ }^{48}$ Many of these eating plans consist of an induction phase and most do not advocate for calorie restriction, based on the premise that the higher protein and fat in the diet, and potentially the circulating ketones, promote satiety. ${ }^{48-50}$ In research and clinical practice, ketosis can be measured as BHB in blood on fingerstick or acetoacetate in urine ${ }^{51}$ Ketone meters are available to monitor for home use and have also been used to follow adherence in studies. ${ }^{52,53}$

These eating patterns have been shown in meta-analysis to cause greater A1c reduction and weight loss than diets without carbohydrate restriction at 3 and 6 months, but this advantage is no longer seen at 12 months. ${ }^{45}$ VLCKDs have shown superior weight loss to other eating plans in some studies ${ }^{54,55}$ and no difference in others. ${ }^{56,57}$ Guldbrand's study compared a low fat diet to a VLCKD and weight loss was similar for the two groups (LFD $-3.99 \pm 4.1 \mathrm{~kg}$; LCD $-4.31 \pm 3.6 \mathrm{~kg}$ ). ${ }^{58}$

Lipid profile changes induced by LCDs can occur in a relatively short time. Additionally, Choi's meta-analysis found that the VLCKD has cardiovascular disease risk reducing effects in patients with diabetes and obesity or overweight, with serum HDL significantly increased and serum triglycerides decreased in those on VLCKD. ${ }^{59}$ Similarly, Westman's 6month trial showed that VLCKD reduced triglycerides and increased HDL, with improvements significantly more than those seen with LFD. ${ }^{60}$

VLCKDs have an immediate impact on glycemic control. Over 3-6 month study periods, Yancy, Saslow, and Westman demonstrated HbAlc improvements in patients on VLCKDs compared to other dietary interventions. ${ }^{34,61,62}$ The success of longer-term interventions has been more modest, but also more difficult to interpret as adherence wanes over time and most studies relied on dietary recall and self-reporting to monitor adherence. 
Medication reductions are a prominent benefit of very low carbohydrate eating patterns. A majority of patients in three to six month VLCKD studies by Yancy and Westman were able to either eliminate or reduce their diabetes medications. ${ }^{34,62}$ Though HbA1c reductions were often modest in studies more than a year in duration, many of these studies still demonstrated significant reductions in insulin and diabetes medication requirements, and it is likely that medication reduction blunted the A1c response. . $^{53,54,63,64}$

Diabetes remission has been defined as achieving an HbAlc of $6.5 \%$ or less in the context of either complete medication cessation or cessation of all medications except metformin. ${ }^{65}$ A systematic review and meta-analysis of studies comparing LCDs to other diets or control arms by Goldenberg identified studies that reported on diabetes remission rates at 6 months ( 8 studies) and 12 months (3 studies). When complete medication independence was not factored in, LCDs achieved greater remission compared to controls at 6 months (an additional 32 per 100 subjects) and more modest remission rates at 12 months (an additional 10 per 100 subjects). When complete medication cessation was used as the definition for diabetes remission, rates were reduced and results were no longer statistically significant. Subgroup analyses showed more significant remission rates at six months among patients on non-insulin agents. ${ }^{42}$

\section{Dietary Adherence Methods and Results in Low Carbohydrate Diet Trials}

Adherence is defined by the World Health Organization as "the extent to which a person's behavior - taking medication, following a diet and/or executing lifestyle changes - corresponds with agreed recommendations from a health care provider" ${ }^{66}$ Assessing dietary adherence remains a challenge due to inconsistencies in how it is measured in studies. One common method is self-reporting using tools such as food records, food frequency questionnaires, or 24-hour dietary recalls. Other measurements include biomarkers, attendance at counseling sessions, and completion rates of the study intervention.

\section{Dietary Recall}

Though dietary recall is commonly used to assess adherence in LCD studies, the validity of self-reported food diaries is widely debated. Kipnis compared biomarkers with dietary recall using Food Frequency Questionnaires and showed that relying on dietary recall can dramatically underestimate intake. ${ }^{67} \mathrm{It}$ is not surprising that underreporting is common given that food records are reactive by nature, 24h dietary recall relies on memory, and food frequency questionnaires have a finite list of foods. In addition to this, a societal stigma against obesity often creates a social desirability bias that potentially exacerbates underreporting. Still, self-reported data can be useful in understanding food behaviors and eating patterns beyond the information provided by biomarkers alone. ${ }^{68}$

Some of the studies already reviewed relied on dietary records to assess adherence. Guldbrand's randomized two-year intervention compared a LFD to VLCKD (Table 1). Diet records were conducted during three consecutive days including one weekend day at 3,6,12, and 24 months, and participants were given dedicated scales to weigh foods. During the first 6 months, adherence to their respective diet was similar between the groups based on mean macronutrient intake, with an increase in energy from fat in the LCD group. The study was unable to conclude that VLCKD caused greater weight reduction. This finding differs from many other studies, but the authors also noted that they used fewer resources to achieve adherence. ${ }^{58}$ Iqbal's randomized two year study of patients with obesity and type 2 diabetes compared an LCD of $<30 \mathrm{~g}$ /day with an LFD. Participants were given educational nutrition sessions carbohydrate counting applications to help maintain their target intake, which was estimated using 24-hour recall. These were group sessions conducted as frequently as weekly in the first month and then gradually decreased to monthly for the study duration. Despite these interventions, adherence was low based on these metrics. Additionally, the VLCKD group had a $60 \%$ attrition rate and participants in both groups appeared to consume similar diets with moderate restriction in carbohydrates at the study's conclusion. ${ }^{69}$ Hu's clinical trial compared a VLCKD with a LFD ( $<30 \%$ fat, $<7 \%$ saturated fat) over 12 -months (Table 1). Overall adherence was measured using a composite score composed of attendance at counselling sessions, deviation from nutrient goals, and urinary ketone presence. Four weekly one-on-one dietician sessions were held in the first month, followed by 10 group sessions every other week for 5 months and 6 monthly group sessions thereafter. Attendance between groups did not vary significantly with over $50 \%$ participating. The study also had a relatively low attrition rate of $21 \%$ in the VLCKD group. However, composite scores for adherence were similar between the groups. A 
Table I Summary of Trials on Adherence to LCKD in Patients with Type 2 Diabetes

\begin{tabular}{|c|c|c|c|c|c|c|c|c|c|c|c|}
\hline Study & Year & $\begin{array}{l}\text { Trial } \\
\text { Design }\end{array}$ & $\begin{array}{l}\text { Study } \\
\text { Duration }\end{array}$ & Arm & $\begin{array}{l}\text { Goal Macronutrient } \\
\text { Intake }\end{array}$ & Dietary Records & $\begin{array}{l}\text { Actual Reported } \\
\text { Macronutrient } \\
\text { Intake at Study } \\
\text { Conclusion }\end{array}$ & Educational Sessions & $\begin{array}{l}\text { Attendance } \\
\text { at } \\
\text { Counseling } \\
\text { Session }\end{array}$ & Attrition & Biomarker Results \\
\hline \multirow[t]{2}{*}{ Guldbrand } & \multirow[t]{2}{*}{2012} & \multirow[t]{2}{*}{$\begin{array}{l}\text { Randomized } \\
\text { trial }\end{array}$} & \multirow[t]{2}{*}{2 years } & $\begin{array}{l}\text { VLCKD } \\
(\mathrm{n}=30)\end{array}$ & $\begin{array}{l}1800 \mathrm{kcal} \text { for men; } 1600 \\
\text { kcal for women with } \\
50 \% \text { of energy from fat, } \\
20 \% \text { energy from } \\
\text { carbohydrates and } 30 \% \\
\text { energy from protein. }\end{array}$ & \multirow{2}{*}{$\begin{array}{l}\text { Obtained at baseline, } 2 \text {, } \\
3,6, \text { and } 12 \text { months. } \\
\text { Records conducted } \\
\text { during three } \\
\text { consecutive days } \\
\text { including one weekend } \\
\text { day and the participants } \\
\text { were provided with } \\
\text { dedicated scales and } \\
\text { notebooks to weigh } \\
\text { and record all food } \\
\text { items consumed during } \\
\text { these periods (no food } \\
\text { frequency } \\
\text { questionnaires) }\end{array}$} & $\begin{array}{l}1251 \pm 425 \mathrm{kcal} ; 31 \% \\
\text { from carbohydrates; } \\
44 \% \text { from fat; } 24 \% \text { from } \\
\text { protein }\end{array}$ & \multirow[t]{2}{*}{$\begin{array}{l}\text { Group meetings about } \\
\text { which food items to } \\
\text { choose given at baseline, } \\
\text { at 2, } 6 \text { and } 12 \text { months by } \\
\text { physicians; no individual } \\
\text { sessions with } \\
\text { nutritionist }\end{array}$} & & $\begin{array}{l}4 \text { did not } \\
\text { attend sessions } \\
\text { and expressed } \\
\text { difficulty with } \\
\text { adherence; } 10 \\
\text { did not } \\
\text { complete study }\end{array}$ & $\begin{array}{l}\text { significant reduction in } \\
\text { Alc from baseline at } 6 \\
\text { months but not at study } \\
\text { conclusion }\end{array}$ \\
\hline & & & & $\begin{array}{l}\text { Low-fat diet } \\
(\mathrm{n}=3 \mathrm{I})\end{array}$ & $\begin{array}{l}1800 \mathrm{kcal} \text { for men; } 1600 \\
\text { kcal for women with } \\
30 \% \text { energy from fat (< } \\
10 \% \text { from saturated fat), } \\
55-60 \% \text { of energy from } \\
\text { carbohydrates and } \\
10-15 \% \text { energy from } \\
\text { protein. }\end{array}$ & & $\begin{array}{l}1458 \pm 45 \mid \mathrm{kcal} ; 47 \% \\
\text { from carbohydrates; } \\
31 \% \text { from fat; } 20 \% \text { from } \\
\text { protein }\end{array}$ & & & $\begin{array}{l}3 \text { did not } \\
\text { attend sessions } \\
\text { and expressed } \\
\text { difficulty with } \\
\text { adherence; } 4 \\
\text { did not } \\
\text { complete study }\end{array}$ & \\
\hline \multirow[t]{2}{*}{ Iqbal } & \multirow[t]{2}{*}{2010} & \multirow[t]{2}{*}{$\begin{array}{l}\text { Randomized } \\
\text { trial }\end{array}$} & \multirow[t]{2}{*}{2 years } & $\begin{array}{l}\text { VLCKD } \\
(n=70)\end{array}$ & $\begin{array}{l}<30 \text { g carbohydrates per } \\
\text { day }\end{array}$ & \multirow{2}{*}{$\begin{array}{l}24 \text { hour self reported } \\
\text { dietary intake at } \\
\text { baseline and 6, 12, and } \\
24 \text { months }\end{array}$} & \multirow[b]{2}{*}{$\begin{array}{l}\text { Self-reported caloric } \\
\text { intake did not differ } \\
\text { significantly between } \\
\text { groups at any time. At } \\
\text { month 24, participants } \\
\text { in the low-carbohydrate } \\
\text { and low-fat groups } \\
\text { reduced their caloric } \\
\text { intake over time by } 397 \\
\text { and } 57 \text { I cal, } \\
\text { respectively. } \\
\text { Macronutrient intake } \\
\text { did not differ } \\
\text { significantly between } \\
\text { groups at any point. }\end{array}$} & \multirow[b]{2}{*}{$\begin{array}{l}\text { Both diet groups were } \\
\text { invited to attend } \\
\text { separate weekly } 2 \mathrm{~h} \\
\text { nutrition classes for the } \\
\text { first month then every } 4 \\
\text { weeks for the study } \\
\text { duration. Sessions } \\
\text { included up to } 10 \\
\text { participants and were } \\
\text { led by a registered } \\
\text { dietitian with expertise } \\
\text { in weight-loss } \\
\text { counseling. Information } \\
\text { was presented by } \\
\text { lecture and handouts } \\
\text { during the first } 30 \text { min of } \\
\text { the session, followed by } \\
\text { reinforcement of } \\
\text { concepts using } \\
\text { interactive games and } \\
\text { quizzes. }\end{array}$} & \multirow[b]{2}{*}{$\begin{array}{l}\text { Participants } \\
\text { attended a } \\
\text { mean (s.d.) of } \\
9.9 \text { (9.5) } \\
\text { sessions, with } \\
\text { a mode of } \\
\text { three sessions } \\
\text { and a median } \\
\text { of six sessions } \\
\text { (interquartile } \\
\text { range 4-12). }\end{array}$} & $\begin{array}{l}60 \% \text { did not } \\
\text { complete study }\end{array}$ & \multirow{2}{*}{$\begin{array}{l}\text { At month } 6 \text {, the low- } \\
\text { carbohydrate group had } \\
\text { a clinically significant } \\
\text { reduction in HbAlc of } \\
-0.5 \% \text { (compared to } \\
-0.1 \% \text { in the low-fat } \\
\text { condition), but this was } \\
\text { not sustained over time }\end{array}$} \\
\hline & & & & $\begin{array}{l}\text { Low-fat diet } \\
(n=74)\end{array}$ & $\begin{array}{l}\text { ( } \leq 30 \% \text { of calories from } \\
\text { fat with a deficit of } 500 \\
\mathrm{kcal} / \mathrm{day} \text { ) }\end{array}$ & & & & & $\begin{array}{l}46 \% \text { did not } \\
\text { complete study }\end{array}$ & \\
\hline
\end{tabular}


Table I (Continued).

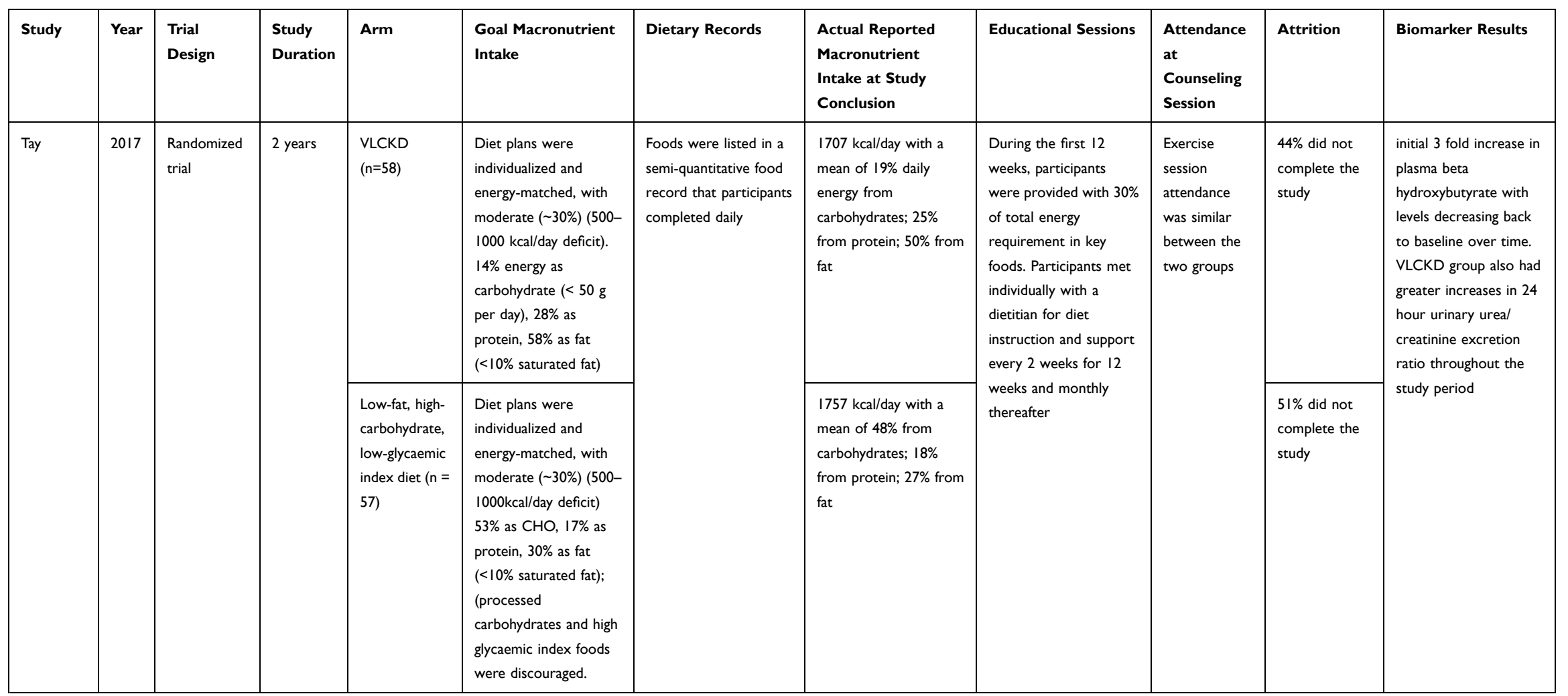




\begin{tabular}{|c|c|c|c|c|c|c|c|c|c|c|}
\hline \multirow[t]{2}{*}{ Yancy } & \multirow[t]{2}{*}{2004} & \multirow[t]{2}{*}{$\begin{array}{l}\text { Randomized } \\
\text { trial }\end{array}$} & \multirow[t]{2}{*}{6 months } & $\begin{array}{l}\text { VLCKD } \\
(n=59)\end{array}$ & $\begin{array}{l}<20 \text { g carbohydrates per } \\
\text { day }\end{array}$ & \multirow{2}{*}{$\begin{array}{l}\text { 24-hour recall of food } \\
\text { intake at baseline and } \\
\text { take-home food } \\
\text { records (5 consecutive } \\
\text { days + weekend) twice } \\
\text { monthly for } 3 \text { months, } \\
\text { then monthly for } 3 \\
\text { months }\end{array}$} & $\begin{array}{l}\mid 461.0 \pm 325.7 \mathrm{kcal} ; 8 \% \\
\text { from carbohydrates ( } 8 \% \\
\text { of daily intake), } 68 \% \\
\text { from fat; } 26 \% \text { from } \\
\text { protein }\end{array}$ & \multirow{2}{*}{$\begin{array}{l}\text { Group meetings took } \\
\text { place at an outpatient } \\
\text { research clinic twice } \\
\text { monthly for } 3 \text { months, } \\
\text { then monthly for } 3 \\
\text { months. These Ihr } \\
\text { meetings consisted of } \\
\text { diet instruction, } \\
\text { supportive counseling, } \\
\text { questionnaires, and } \\
\text { biomedical } \\
\text { measurements }\end{array}$} & $\begin{array}{l}24 \% \text { did not } \\
\text { complete the } \\
\text { study }\end{array}$ & \multirow{2}{*}{$\begin{array}{l}86 \% \text { of VLCKD had } \\
\text { trace or greater urinary } \\
\text { ketones at } 2 \text { weeks and } \\
\text { decreased to } 42 \% \text { at } 24 \\
\text { weeks.64\% of VLCKD } \\
\text { had moderate or } \\
\text { greater ketones and } \\
\text { decreased to } 18 \% \text { at } 24 \\
\text { weeks. VLCKD had } \\
\text { statistically greater } \\
\text { changes in TG, HDL, } \\
\text { and ratio of TG to } \\
\text { HDL. }\end{array}$} \\
\hline & & & & $\begin{array}{l}\text { Low-fat diet } \\
(\mathrm{n}=60)\end{array}$ & $\begin{array}{l}<30 \% \text { energy from fat, } \\
<200 \mathrm{mg} \text { of cholesterol } \\
\text { daily, and deficit of } \\
500-1000 \mathrm{kcal} / \mathrm{d}\end{array}$ & & $\begin{array}{l}1502.0 \pm 162.1 \mathrm{kcal} ; \\
52 \% \text { from } \\
\text { carbohydrates, } 29 \% \\
\text { from fat, } 19 \% \text { from } \\
\text { protein }\end{array}$ & & $\begin{array}{l}43 \% \text { did not } \\
\text { complete the } \\
\text { study }\end{array}$ & \\
\hline \multirow[t]{2}{*}{ Westman } & \multirow[t]{2}{*}{2008} & \multirow[t]{2}{*}{$\begin{array}{l}\text { Randomized } \\
\text { trial }\end{array}$} & \multirow[t]{2}{*}{6 months } & $\begin{array}{l}\text { VLCKD } \\
(n=48)\end{array}$ & $\begin{array}{l}<20 \text { g carbohydrates per } \\
\text { day }\end{array}$ & \multirow{2}{*}{$\begin{array}{l}\text { Food records ( } 5 \\
\text { consecutive days, } \\
\text { including a weekend) at } \\
\text { baseline and weeks } 4 \text {, } \\
12 \text {, and } 24\end{array}$} & $\begin{array}{l}1550 \pm 440 \mathrm{kcal} ; 13 \% \\
\text { from carbohydrates, } \\
59 \% \text { from fat, } 28 \% \text { from } \\
\text { protein }\end{array}$ & \multirow{2}{*}{$\begin{array}{l}\text { Group meetings took } \\
\text { place every week for } 3 \\
\text { months, then every } \\
\text { other week for } 3 \\
\text { months. These } \\
\text { consisted of physician } \\
\text { review of BP and BG } \\
\text { medications if } \\
\text { applicable. }\end{array}$} & $\begin{array}{l}56 \% \text { did not } \\
\text { complete the } \\
\text { study }\end{array}$ & \multirow{2}{*}{$\begin{array}{l}\text { Mean change in HbAlc } \\
\text { for LCKD group was } \\
-1.5 \% \text {, significantly } \\
\text { more than LGID with } \\
\text { HbAIc change of } \\
-0.5 \%\end{array}$} \\
\hline & & & & $\begin{array}{l}\text { Low glycemic } \\
\text { index diet } \\
(n=49)\end{array}$ & $\begin{array}{l}55 \% \text { of daily energy } \\
\text { intake from } \\
\text { carbohydrate with }-500 \\
\text { kcal less than calculated } \\
\text { energy intake for } \\
\text { weight maintenance }\end{array}$ & & $\begin{array}{l}1335 \pm 372 \mathrm{kcal} \text { per } \\
\text { day; } 44 \% \text { from } \\
\text { carbohydrates, } 36 \% \\
\text { from fat, } 20 \% \text { from } \\
\text { protein }\end{array}$ & & $\begin{array}{l}37 \% \text { did not } \\
\text { complete the } \\
\text { study }\end{array}$ & \\
\hline
\end{tabular}

(Continued) 
Table I (Continued).

\begin{tabular}{|c|c|c|c|c|c|c|c|c|c|c|c|}
\hline Study & Year & $\begin{array}{l}\text { Trial } \\
\text { Design }\end{array}$ & $\begin{array}{l}\text { Study } \\
\text { Duration }\end{array}$ & Arm & $\begin{array}{l}\text { Goal Macronutrient } \\
\text { Intake }\end{array}$ & Dietary Records & $\begin{array}{l}\text { Actual Reported } \\
\text { Macronutrient } \\
\text { Intake at Study } \\
\text { Conclusion }\end{array}$ & Educational Sessions & $\begin{array}{l}\text { Attendance } \\
\text { at } \\
\text { Counseling } \\
\text { Session }\end{array}$ & Attrition & Biomarker Results \\
\hline \multirow[t]{2}{*}{$\mathrm{Hu}$} & \multirow[t]{2}{*}{2016} & \multirow[t]{2}{*}{$\begin{array}{l}\text { Randomized } \\
\text { trial }\end{array}$} & \multirow[t]{2}{*}{$\begin{array}{l}12 \\
\text { months }\end{array}$} & $\begin{array}{l}\text { VLCKD } \\
(\mathrm{n}=75)\end{array}$ & $\begin{array}{l}<40 \mathrm{~g} \text { carbohydrates per } \\
\text { day }\end{array}$ & \multirow[t]{2}{*}{$\begin{array}{l}\text { Two } 24 \mathrm{~h} \text { dietary recalls, } \\
\text { one on a week day and } \\
\text { thet other on a } \\
\text { weekend day, were } \\
\text { obtained from each } \\
\text { participant by a } \\
\text { certified dietician at } 0 \text {, } \\
3,6 \text {, and } 12 \text { months }\end{array}$} & $\begin{array}{l}73.9 \%, 59.7 \% \text {, and } \\
44.8 \% \text { met } \\
\text { carbohydrate goals at 3, } \\
6 \text {, and } 12 \text { months. } \\
\text { Those who did not had } \\
\text { mean deviations of } \\
145 \% \text { (representing a } \\
\text { carbohydrate intake of } \\
\text { approximately } 98 \mathrm{gd} \\
-1), 104 \%(82 \mathrm{gd}-1) \\
\text { and } 198 \%(119 \mathrm{gd}-1) \text {, } \\
\text { respectively }\end{array}$ & \multirow[t]{2}{*}{$\begin{array}{l}20 \text { regular dietary } \\
\text { counselling sessions } \\
\text { including four weekly } \\
\text { individual sessions for } \\
\text { the first month followed } \\
\text { by } 10 \text { group sessions } \\
\text { every other week for } 5 \\
\text { months and } 6 \text { monthly } \\
\text { group sessions after. }\end{array}$} & \multirow[t]{2}{*}{$\begin{array}{l}\text { There was no } \\
\text { significant } \\
\text { difference in } \\
\text { attendance } \\
\text { between } \\
\text { groups. } 56.7 \% \\
\text { of VLCKD } \\
\text { and 52.3\% of } \\
\text { low-fat diet } \\
\text { group } \\
\text { attended } \\
\text { counselling } \\
\text { sessions. }\end{array}$} & $\begin{array}{l}21 \% \text { did not } \\
\text { complete the } \\
\text { study }\end{array}$ & \multirow[t]{2}{*}{$\begin{array}{l}\text { VLCKD group had } \\
\text { higher cumulative } \\
\text { percentage urine } \\
\text { ketones at } 3,6 \text {, and I2 } \\
\text { months compared to } \\
\text { low-fat diet group. This } \\
\text { was associated with } \\
\text { greater reductions in } \\
\text { body weight and } \\
\text { percent fat mass and } \\
\text { increase in percent lear } \\
\text { mass. No associations } \\
\text { were identified in the } \\
\text { low-fat group. }\end{array}$} \\
\hline & & & & $\begin{array}{l}\text { Low-fat diet } \\
(\mathrm{n}=73)\end{array}$ & $\begin{array}{l}\text { Total fat }<30 \% \text { of daily } \\
\text { energy and saturated fat } \\
<7 \% \text { of daily energy }\end{array}$ & & $\begin{array}{l}59.4 \% \text { and } 42.2 \% \text { of } \\
\text { individuals met total } \\
\text { and saturated fat goals } \\
\text { at } 3 \text { months, } 64.8 \% \text { and } \\
33.3 \% \text { did at } 6 \text { months } \\
\text { and } 55.6 \% \text { and } 27.8 \% \\
\text { did at } 12 \text { months, } \\
\text { respectively. Those who } \\
\text { consumed more than } \\
\text { the goal had mean } \\
\text { deviations of } 19.1 \% \\
\text { (representing an intake } \\
\text { of about } 35.7 \% \text { of daily } \\
\text { energy from total fat) } \\
\text { and } 40.6 \% \text { (9.8\% } \\
\text { saturated fat) at } 3 \\
\text { months, } 17.4 \% \text { (35.2\% } \\
\text { total fat) and } 39.8 \% \\
(9.8 \% \text { saturated fat) at } \\
6 \text { months and } 23.3 \% \\
\text { (37.0\% total fat) and } \\
46.2 \% \text { (10.2\% saturated } \\
\text { fat) at } 12 \text { months, } \\
\text { respectively }\end{array}$ & & & $\begin{array}{l}18 \% \text { did not } \\
\text { complete the } \\
\text { study }\end{array}$ & \\
\hline
\end{tabular}




\begin{tabular}{|c|c|c|c|c|c|c|c|c|c|c|c|}
\hline Saslow & 2017 & $\begin{array}{l}\text { Randomized } \\
\text { trial }\end{array}$ & $\begin{array}{l}12 \\
\text { months }\end{array}$ & $\begin{array}{l}\text { VLCKD } \\
(n=16)\end{array}$ & $\begin{array}{l}\text { Carbohydrate intake } \\
\text { reduced over } 7-10 \text { days } \\
\text { to between 20-50 } \\
\text { grams of carbohydrates } \\
\text { a day, not including } \\
\text { fiber, with the goal of } \\
\text { achieving nutritional } \\
\text { ketosis as measured by } \\
\text { blood beta- } \\
\text { hydroxybutyrate level } \\
0.5-3 \mathrm{mM} \text { twice a week } \\
\text { at home. Protein intake } \\
\text { prior to the study was } \\
\text { maintained and the rest } \\
\text { of the calories were } \\
\text { derived from fat. }\end{array}$ & $\begin{array}{l}\text { Food intake was } \\
\text { assessed with an online } \\
\text { 24- hour food recall } \\
\text { questionnaire. Timing } \\
\text { of questionnaire } \\
\text { administration not } \\
\text { reported. }\end{array}$ & $\begin{array}{l}1693.7 \mathrm{kcal} ; 57.8 \mathrm{~g} \text { from } \\
\text { carbohydrates; } 24.2 \% \text { of } \\
\text { daily calorie intake from } \\
\text { protein; } 58.0 \% \text { of } \\
\text { dietary intake from fat }\end{array}$ & $\begin{array}{l}\text { Participants attended } 19 \\
\text { classes over } 12 \text { months } \\
\text { including twelve 2-hour } \\
\text { classes that met weekly } \\
\text { initially and gradually } \\
\text { tapered to } 1.5 \text {-h every } 2 \\
\text { months. One hour was } \\
\text { devoted to instruction } \\
\text { on the assigned diet, } \\
\text { with three classes also } \\
\text { discussing the } \\
\text { importance of sleep and } \\
\text { exercise. Each class } \\
\text { session included a break } \\
\text { with snacks appropriate } \\
\text { to the assigned diet. } \\
\text { Participants were } \\
\text { encouraged to change } \\
\text { their diet gradually; } \\
\text { ideally, by the fourth } \\
\text { class, participants were } \\
\text { to have changed all of } \\
\text { their meals to be in } \\
\text { alignment with the new } \\
\text { recommendations. } \\
\text { Those on VLCKD were } \\
\text { given a goal of B-OH } \\
\text { between } 0.5 \text { and } \\
3 \text { mmol measured twice } \\
\text { weekly at home. Half of } \\
\text { each two-hour class in } \\
\text { both groups was } \\
\text { focused on learning } \\
\text { skills to support } \\
\text { behavior change and } \\
\text { diet maintenance. }\end{array}$ & Not reported & $\begin{array}{l}\text { 14/16 (87.5\%); } \\
\text { similar } \\
\text { between } \\
\text { groups }\end{array}$ & $\begin{array}{l}\text { HbAIc in VLCKD } \\
\text { group improved from } \\
\text { baseline } 6.6 \% \text { to } 6.0 \% \\
\text { and } 6.1 \% \text { at } 6 \text { and I2 } \\
\text { months, respectively. } \\
\text { HbAIc in MCCR group } \\
\text { improved from } 6.9 \% \text { at } \\
\text { baseline to } 6.7 \% \text { at } 6 \\
\text { and } 12 \mathrm{~m} \text {. Body weight } \\
\text { ( } \mathrm{kg} \text { ) decreased } 7 \mathrm{~kg} \\
\text { (from } 99.9 \mathrm{~kg} \text { to } 92.0 \\
\mathrm{~kg} \text { ) in VLCKD group } \\
\text { compared to a decrease } \\
\text { of } 2 \mathrm{~kg} \text { (from } 97.5 \text { to } \\
95.8 \mathrm{~kg} \text { ) in MCCR } \\
\text { group at I2m. With } \\
\text { regards to lipids, TG } \\
\text { improved from } 102.6 \text { to } \\
92.7 \mathrm{mg} / \mathrm{dL} \text { in VLCKD } \\
\text { vs an increase from } \\
158.9 \text { to } 173.4 \mathrm{mg} / \mathrm{dL} \text { in } \\
\text { MCCR group. Changes } \\
\text { in HDL were not } \\
\text { significant. }\end{array}$ \\
\hline
\end{tabular}

(Continued) 
Table I (Continued).

\begin{tabular}{|c|c|c|c|c|c|c|c|c|c|c|c|}
\hline Study & Year & $\begin{array}{l}\text { Trial } \\
\text { Design }\end{array}$ & $\begin{array}{l}\text { Study } \\
\text { Duration }\end{array}$ & Arm & $\begin{array}{l}\text { Goal Macronutrient } \\
\text { Intake }\end{array}$ & Dietary Records & $\begin{array}{l}\text { Actual Reported } \\
\text { Macronutrient } \\
\text { Intake at Study } \\
\text { Conclusion }\end{array}$ & Educational Sessions & $\begin{array}{l}\text { Attendance } \\
\text { at } \\
\text { Counseling } \\
\text { Session }\end{array}$ & Attrition & Biomarker Results \\
\hline & & & & $\begin{array}{l}\text { Medium } \\
\text { carbohydrate, } \\
\text { low fat, } \\
\text { calorie } \\
\text { restricted, } \\
\text { carbohydrate } \\
\text { counting diet } \\
(\mathrm{n}=18)\end{array}$ & $\begin{array}{l}\text { 45-50\% of calories } \\
\text { from carbohydrates; } \\
\text { carbohydrates counted } \\
\text { using } 15 \text { grams as a unit. } \\
\text { Most participants were } \\
\text { asked to eat } 3 \\
\text { carbohydrate units per } \\
\text { meal and I per snack, } \\
\text { or } 165 \text { grams of } \\
\text { carbohydrates a day. } \\
\text { Protein intake was kept } \\
\text { the same as before the } \\
\text { study and fat } \\
\text { consumption was } \\
\text { lowered. Participants } \\
\text { ate } 500 \text { fewer } \\
\text { kilocalories (kcal) per } \\
\text { day than their } \\
\text { calculated maintenance } \\
\text { needs based on their } \\
\text { age, weight, height, and } \\
\text { physical activity level, } \\
\text { using the formula from } \\
\text { the Institute of } \\
\text { Medicine Dietary } \\
\text { Reference Guidelines }\end{array}$ & & $\begin{array}{l}\text { 1380.8 kcal per day; } \\
138.5 \mathrm{~g} \text { carbohydrates; } \\
20.5 \% \text { from protein; } \\
35.1 \% \text { from fat }\end{array}$ & & & $\begin{array}{l}15 / 18(83.3 \%) ; \\
\text { similar } \\
\text { between } \\
\text { groups }\end{array}$ & $\begin{array}{l}\text { There was no } \\
\text { statistically significant } \\
\text { change in blood } \\
\text { glucose. }\end{array}$ \\
\hline
\end{tabular}




\begin{tabular}{|c|c|c|c|c|c|c|c|c|c|c|c|}
\hline \multirow[t]{2}{*}{ Mayer } & \multirow[t]{2}{*}{2014} & \multirow[t]{2}{*}{$\begin{array}{l}\text { Randomized } \\
\text { trial }\end{array}$} & \multirow[t]{2}{*}{48 weeks } & $\begin{array}{l}\text { VLCKD } \\
(n=22)\end{array}$ & $\begin{array}{l}\text { Daily carbohydrate } \\
\text { intake limited to less } \\
\text { than } 20 \mathrm{~g} \text {, but calories } \\
\text { were not restricted. } \\
\text { Carbohydrate intake } \\
\text { was slowly liberalized if } \\
\text { participants approached } \\
\text { their goal weight or } \\
\text { cravings threatened } \\
\text { adherence. }\end{array}$ & \multirow[t]{2}{*}{$\begin{array}{l}\text { Diet adherence was } \\
\text { measured using 4-day } \\
\text { food records (including } \\
2 \text { weekend days) at } \\
\text { baseline and weeks 2, } \\
\text { 12, 24, 36, and } 48\end{array}$} & $\begin{array}{l}\text { Mean daily } \\
\text { carbohydrate intake } \\
\text { was } 75.9 \mathrm{~g} \text {, total fat } \\
103.2 \mathrm{~g} \text {, and energy } \\
1707.9 \mathrm{kcal} / \text { day }\end{array}$ & \multirow[t]{2}{*}{$\begin{array}{l}\text { Small group meetings ( } 6 \\
\text { to } 12 \text { participants) every } \\
2 \text { weeks for } 24 \text { weeks, } \\
\text { then every } 4 \text { weeks for } \\
24 \text { weeks. Meetings } \\
\text { lasted I to } 2 \text { hours and } \\
\text { consisted of study } \\
\text { measurements followed } \\
\text { by group counseling that } \\
\text { were parallel between } \\
\text { the } 2 \text { interventions but } \\
\text { specific to diet. }\end{array}$} & \multirow[t]{2}{*}{ Not reported } & $\begin{array}{l}50 \% \text { of patients } \\
\text { did not have } \\
\text { food records } \\
\text { at the end of } \\
\text { the study } \\
\text { period }\end{array}$ & $\begin{array}{l}\text { Fasting glucose declined } \\
\text { from mean } 152.6 \text { to } \\
133.7(p=0.2)\end{array}$ \\
\hline & & & & $\begin{array}{l}\text { Low Fat Diet } \\
+ \text { Orlistat } \\
(n=24)\end{array}$ & $\begin{array}{l}\text { Daily intake of fat } \\
\text { limited to }<30 \% \text { energy, } \\
\text { saturated fat to }<10 \% \\
\text { energy, cholesterol to } \\
<300 \mathrm{mg} \text {, and calories } \\
\text { ( } 500-1000 \mathrm{kcal} \text { deficit). } \\
\text { Orlistat } 120 \mathrm{mg} \text { was } \\
\text { taken three times per } \\
\text { day. }\end{array}$ & & $\begin{array}{l}\text { Mean daily } \\
\text { carbohydrate intake } \\
\text { was } 155.8 \mathrm{~g} \text {, total fat } \\
55.5 \mathrm{~g} \text {, and energy } \\
1419.6 \mathrm{kcal} / \mathrm{day}\end{array}$ & & & $\begin{array}{l}50 \% \text { of patients } \\
\text { did not have } \\
\text { food records } \\
\text { at the end of } \\
\text { the study } \\
\text { period }\end{array}$ & $\begin{array}{l}\text { Fasting glucose declined } \\
\text { from mean } 149.0 \text { to } \\
\mid 46.8\end{array}$ \\
\hline \multirow[t]{2}{*}{ Davis } & \multirow[t]{2}{*}{2009} & \multirow[t]{2}{*}{$\begin{array}{l}\text { Randomized } \\
\text { trial }\end{array}$} & \multirow[t]{2}{*}{$\begin{array}{l}12 \\
\text { months }\end{array}$} & $\begin{array}{l}\text { VLCKD } \\
(n=55)\end{array}$ & $\begin{array}{l}\text { Initially } 20-25 \mathrm{~g} \text { of } \\
\text { carbohydrate } \times 2 \\
\text { weeks. As weight } \\
\text { reduced, carbohydrate } \\
\text { intake was increased at } \\
5 \mathrm{~g} \text { per week. }\end{array}$ & \multirow{2}{*}{$\begin{array}{l}\text { Single-day } 24 \mathrm{~h} \text { recall by } \\
\text { in-person interviews } \\
\text { were obtained at } \\
\text { baseline, } 6 \text {, and } 12 \\
\text { months. At } 3 \text { months, } \\
\text { food diaries were } \\
\text { reviewed for the day } \\
\text { prior. }\end{array}$} & $\begin{array}{l}1642 \pm 600 \mathrm{kcal} ; 33 \% \text { of } \\
\text { calories from } \\
\text { carbohydrates; } 44 \% \\
\text { from fat, } 23 \% \text { from } \\
\text { protein }\end{array}$ & \multirow{2}{*}{$\begin{array}{l}\text { All received } 45 \text { min } \\
\text { dietary instruction of } \\
\text { registered dietician and } \\
\text { were given a specific } \\
\text { gram allowance of } \\
\text { carbohydrates or fat to } \\
\text { achieve a I-pound } \\
\text { weight loss each week. } \\
\text { They also had a total of } \\
\text { six scheduled, 30-min } \\
\text { visits with the dietician } \\
\text { for additional counseling } \\
\text { over I2 months. } \\
\text { Participants also had } \\
\text { study visits I-2x weekly } \\
\text { for the Ist month } \\
\text { followed by every } 6 \\
\text { weeks in addition to this } \\
\text { to review dietary } \\
\text { adherence and adjust } \\
\text { medications. }\end{array}$} & Not reported & $\begin{array}{l}\text { No difference } \\
\text { between arms. } \\
8 \text { withdrew. }\end{array}$ & \multirow{2}{*}{$\begin{array}{l}\text { Decreased adherence } \\
\text { noted based on } \\
\text { macronutrient intake } \\
\text { over } 12 \text { months. } \\
\text { Weight reduction was } \\
\text { the same in both groups } \\
\text { at I year. There was no } \\
\text { significant differences in } \\
\text { Alc or lipids at I year. }\end{array}$} \\
\hline & & & & $\begin{array}{l}\text { Low-fat diet } \\
(\mathrm{n}=50)\end{array}$ & $\begin{array}{l}\text { Fat intake }<25 \% \text { of } \\
\text { energy needs, based on } \\
\text { baseline weight. }\end{array}$ & & $\begin{array}{l}1810 \pm 590 \mathrm{kcal} ; 50 \% \text { of } \\
\text { calories from } \\
\text { carbohydrates; } 31 \% \\
\text { from fat, } 19 \% \text { from } \\
\text { protein }\end{array}$ & & Not reported & 6 withdrew. & \\
\hline
\end{tabular}


Table I (Continued)

\begin{tabular}{|c|c|c|c|c|c|c|c|c|c|c|c|}
\hline Study & Year & $\begin{array}{l}\text { Trial } \\
\text { Design }\end{array}$ & $\begin{array}{l}\text { Study } \\
\text { Duration }\end{array}$ & Arm & $\begin{array}{l}\text { Goal Macronutrient } \\
\text { Intake }\end{array}$ & Dietary Records & $\begin{array}{l}\text { Actual Reported } \\
\text { Macronutrient } \\
\text { Intake at Study } \\
\text { Conclusion }\end{array}$ & Educational Sessions & $\begin{array}{l}\text { Attendance } \\
\text { at } \\
\text { Counseling } \\
\text { Session }\end{array}$ & Attrition & Biomarker Results \\
\hline \multirow[t]{2}{*}{ Yancy } & \multirow[t]{2}{*}{2020} & \multirow[t]{2}{*}{$\begin{array}{l}\text { Randomized } \\
\text { trial }\end{array}$} & \multirow[t]{2}{*}{48 weeks } & $\begin{array}{l}\text { VLCKD } \\
\text { weight } \\
\text { management } \\
(n=127)\end{array}$ & $\begin{array}{l}<20-30 \mathrm{~g} \text { of } \\
\text { carbohydrate/d initially } \\
\text { followed by an increase } \\
\text { in carbohydrates based } \\
\text { on individual }\end{array}$ & \multirow[t]{2}{*}{$\begin{array}{l}\text { Food records were } \\
\text { collected at baseline } \\
\text { and every } 16 \text { weeks by } \\
\text { 3-day food records. }\end{array}$} & Not reported. & $\begin{array}{l}\text { Every } 2 \text { weeks } \times 16 \\
\text { weeks followed by every } \\
8 \text { weeks thereafter. } \\
\text { More comprehensive } \\
\text { classes including low- } \\
\text { carbohydrate nutrition, } \\
\text { physical activity, and } \\
\text { weight management } \\
\text { counseling, as well as } \\
\text { dietician-led nutritional } \\
\text { counseling. }\end{array}$ & \multirow[t]{2}{*}{$\begin{array}{l}60.6 \% \text { of } \\
\text { VLCKD } \\
\text { weight } \\
\text { management } \\
\text { group and } \\
55.2 \% \text { of } \\
\text { medication } \\
\text { intensification } \\
\text { arm attended } \\
\text { at least } 75 \% \text { of } \\
\text { counseling } \\
\text { sessions. }\end{array}$} & $\begin{array}{l}14.2 \% \text { did not } \\
\text { complete } \\
\text { HbAlc } \\
\text { measurements } \\
\text { at study } \\
\text { conclusion. }\end{array}$ & \multirow[t]{2}{*}{$\begin{array}{l}\text { VLCKD group was } \\
\text { noninferior but not } \\
\text { superior compared to } \\
\text { medication } \\
\text { intensification group. } \\
\text { VLCKD group had } \\
\text { greater mean reduction } \\
\text { in Alc at } 16 \text { weeks but } \\
\text { not at } 48 \text { weeks. }\end{array}$} \\
\hline & & & & $\begin{array}{l}\text { Medication } \\
\text { intensification } \\
\operatorname{arm}(n=136)\end{array}$ & Not specified. & & Not reported. & $\begin{array}{l}\text { Every } 4 \text { weeks } \times 16 \\
\text { weeks followed by every } \\
8 \text { weeks thereafter. } \\
\text { Nurse-led classes } \\
\text { focused on DM } \\
\text { management. }\end{array}$ & & $\begin{array}{l}14 \% \text { did not } \\
\text { complete } \\
\text { HbAlc } \\
\text { measurements } \\
\text { at study } \\
\text { conclusion. }\end{array}$ & \\
\hline
\end{tabular}


one-interquartile-range increase in composite score suggested better adherence to VLCKD and was associated with greater weight loss and improved body composition with increased lean mass. Indicators of adherence in the LFD were not associated with weight loss. ${ }^{70}$

The results of these studies demonstrate the challenges of dietary adherence and of measuring adherence with dietary recall.

\section{Biomarkers}

Other studies have used a combination of biomarkers with self-reporting to address these limitations. Tay assessed dietary intake using daily weighed food records with software to calculate average quarterly nutrient intake between VLCKD and LFD (Table 1). Biomarkers were used to confirm adherence: protein intake was measured using 24-hour urinary urea to creatinine ratio and carbohydrate reduction by using monthly plasma BHB concentrations. Plasma BHB concentrations and the ratio of urinary urea to creatinine excretion increased and remained higher over the 52-week period than with the high carbohydrate diet, consistent with lower carbohydrate and higher protein intake in LCD patients. In addition to individualized dietary plans, this study involved one-on-one sessions with a dietician for dietary instruction and support every 2 weeks for the first 12 weeks and monthly thereafter. The use of individualized dietary sessions may have played a part in the high adherence rate noted. ${ }^{71}$ Yancy's unblinded study compared VLCKD versus orlistat plus LFD (Table 1). As participants approached their goal weight or if cravings threatened dietary adherence, $5 \mathrm{~g}$ of carbohydrates were added to their daily intake each week until weight was maintained or cravings diminished. ${ }^{55}$ Dietary adherence was measured using urinary ketones in combination with 4-day food records at baseline and at 2, 12, $24,36$, and 48 weeks. The proportion of VLCKD participants with urinary ketones present ( $\geq 5 \mathrm{mg} / \mathrm{dL}[\geq 0.9 \mathrm{mmol} / \mathrm{L}])$ at two weeks was $72 \%$. This declined to a low of $13 \%$ at 48 weeks; the decline could have been due to decreased adherence, increased carbohydrate intake for weight maintenance or increased use of ketones for energy leading to lower urinary levels. Participants had one-hour group meetings for dietary instruction and counseling twice monthly for 3 months then monthly for 3 months. The frequency of these sessions may have contributed to the relatively low attrition rate of $24 \%$ in the VLCKD group. ${ }^{63}$

Similarly, Goldstein's study comparing a VLCKD with the calorie restricted ADA diet used urinary ketones measured at 6 weeks and 3, 6, and 12 months to compliment the information obtained by 3 day dietary recall. The authors noted that mean carbohydrate intake at 3,6 and 12 months was more than double the goal. This correlated with urinary ketone elevation in $61 \%$ of the VLCKD diet at 6 week after diet initiation, but in only $7 \%$ at the conclusion of the study 12 months later (Goldstein et al, 2011).

Westman's study randomized individuals with obesity and type 2 diabetes to either a VLCKD or a low-glycemic, reduced calorie diet $(500 \mathrm{kcal} /$ day deficit from weight maintenance diet) (Table 1). Adherence to diet and exercise was measured using a combination of self-report, food records, and urinary ketones. All participants completed food records (on 5 consecutive days, including a weekend) at baseline and at weeks 4, 12, and 24. Though group rather than individual meetings were held, these were conducted as frequently as weekly for 3 months and included a physician. After that, regular meetings were held every other week for 3 months. Though only $58 \%$ of participants completed the study, both groups had good adherence based on self reported dietary intake and interventions led to improvements in hemoglobin $\mathrm{A}_{1 \mathrm{c}}$, fasting glucose, fasting insulin, and weight loss, though the VLCKD group had greater improvements in hemoglobin $\mathrm{A}_{1 \mathrm{c}}$, body weight, and HDL. ${ }^{37}$

\section{Challenges Affecting LCD Adherence in Patients with Diabetes}

\section{Cultural Barriers}

Although it is well-established that genetics influences the development and degree of complications associated with type 2 diabetes, it is also known that behavioral factors including diet and physical activity are directly associated with diabetes diagnosis and progression. Dietary habits are shaped early in life and are influenced by an individual's knowledge and experiences which are often shaped by their culture. ${ }^{72}$ Today, there is increased recognition for the impact of culture on health. ${ }^{73}$ Culture refers to shared values, beliefs and social behaviors that collectively shape a 
group's identity and interaction in and with their environment. ${ }^{73}$ When prescribing diabetes management plans, it is important that clinicians are sensitive to their patients' cultural traditions so that counseling and interventions are appropriate and more likely to have meaningful outcomes. ${ }^{74}$

East Asian diets are typically white rice-based with less consumption of whole grains and fat. Excess consumption of white rice has been associated with higher risk of type 2 diabetes due to its ${ }^{75}$ high glycemic index and load, leading to postprandial blood glucose excursions and hyperinsulinemia. ${ }^{76}$ Zhao et al recently analyzed gender difference in dietary energy of Chinese adults and found that the intake of carbohydrates was $282.4 \mathrm{~g} /$ day in males and $242 \mathrm{~g} /$ day in females, respectively. ${ }^{77}$ Data from the 2013-2015 Korean National Health and Nutrition Examination Survey found that a significant part of the population exceeded recommended ranges of carbohydrates. A higher carbohydrate diet was associated with lower intake of energy and saturated fats, including more grains and fruit but less meat, fish, eggs, beans, and dairy. ${ }^{78}$ Noodles are often used as the main ingredient in Korean meals and are additionally noted to have high glycemic loads and are usually eaten in larger than recommend serving sizes ${ }^{79}$ Similarly, a typical Thai meal involves many complementary dishes, which are served together with rice ${ }^{80}$ The importance of rice is reflected by one of the most common greetings, "kin khaao reuu yang?" (Have you consumed rice yet?). ${ }^{81}$

Traditional South Asian diets are often high in starchy foods such as potatoes, flatbread (roti and chapati), rice and fried snacks. ${ }^{82,83}$ The STARCH study, a cross-sectional multicenter survey of 796 patients, demonstrated carbohydrates made up over $60 \%$ of energy intake for Indian patients with T2DM. ${ }^{84}$ Due to globalization, industrialization and socioeconomic changes, dietary patterns in South Asia have generally shifted to consumption of high fat, less nutrient-dense foods with increased intake of sugar and dairy, particularly among urban and higher income rural dwellers. ${ }^{85}$ Both the traditional eating patterns and modern eating patterns can make adherence to dietary patterns difficult in South Asian countries. This is of particular importance given that South Asians develop type 2 diabetes at younger ages and at lower BMI and have increased rates of complications. ${ }^{82}$ A systematic review noted that for individuals of South Asian descent, food plays a key role in maintaining relationships. This often resulted in $^{74}$ social pressure to not adhere to diabetic diets when at social events. ${ }^{74}$

Traditional Hispanic diets among Dominican, Mexican and Puerto Rican communities include staples such as rice, beans and tortillas. ${ }^{86}$ As seen in other cultures, migration is associated with changes in dietary habits. Mexican Americans are one of the largest growing minority groups in the USA. Higher acculturation among adults of Mexican descent living in the USA has been associated with lower intake of the healthy foods in a traditional Mexican diet, including fruits, vegetables, legumes, and whole grains. Instead, adoption of a Western diet is common, which is usually low in fruits and vegetables and high in refined grains and added sugar. ${ }^{87-89}$ The 2017-2018 prevalence of diagnosed diabetes in adults aged 18 years or older living in the US was highest among people of Hispanic origin (12.5\%), and non-Hispanic blacks (11.7\%). Among adults of Hispanic origin, Mexicans (14.4\%) and Puerto Ricans (12.4\%) had the highest prevalence, followed by Central/South Americans (8.3\%) and Cubans (6.5\%) (Prevalence of Diagnosed Diabetes | Diabetes | CDC).

In the US, non-Hispanic blacks (NHBs) are comprised of heterogeneous groups including those from African and Caribbean ancestry. Despite differences in ethnicity, both groups of NHBs in the US are more likely to be diagnosed with diabetes and experience higher rates of complications and mortality compared to their Caucasian counterparts. ${ }^{90}$ Eating patterns of NHBs are shaped by historical factors such as the transatlantic slave trade, and the common social belief that "more shapely" body types are deemed more desirable compared to other cultures' value of "ideal thinness". Traditional African America cuisine, often referred to as "soul food" includes starchy vegetables and legumes (blackeyed peas, pinto beans, lima beans), grains (rice, grits, cornbread, biscuits), a variety of green leafy vegetables (collards, mustard and turnip greens) in addition to meats that are often breaded and fried. It is not uncommon to see combinations of foods such as rice paired with black eyed peas ("hopping john") or red beans and rice. It is also common to see smoked meats added to vegetables to increase flavor. ${ }^{86} \mathrm{~A}$ common theme of qualitative and ethnographic studies investigating eating practices of African Americans is the importance of taste and concerns that healthier options did not taste as satisfying as traditional foods. ${ }^{92-95}$ Social events with more attendees usually translated to more traditional cultural foods being present and increased the likelihood of choosing less healthy options and overeating. ${ }^{92}$ 
Because of vast similarities in culture such as religion and language, the Middle Eastern and North African (MENA) Region are often grouped together when describing health outcomes. Traditionally, this region's diet was felt to be one of the healthiest as it is the basis of the well-studied "Mediterranean diet" that is high in vegetable proteins, fibers, minerals and vitamins. The cultural emphasis on these foods makes it challenging for patients to adhere to ketogenic diets. The authors of the Goldstein study noted difficulty involved in prescribing the Atkins diet over an extended period in the Mediterranean area, where fruit and vegetable intake is high. Patients were able to avoid eating "pure carbohydrates" (bread, rice, etc.), but continued to consume vegetables and dairy products. ${ }^{96}$ Over the past few decades, ${ }^{97}$ many countries in this region continue to experience a socioeconomic transition due to urbanization, and this has impacted nutritional choices. There has been a shift away from tradition to a diet heavier in processed foods, ${ }^{98}$ sugar sweetened beverages, and often lacking in vegetables, fruits, and whole grains. ${ }^{99}$ Mean energy intake in most countries in this region is higher than the global average, with Turkey and Yemen ranking among the highest. ${ }^{98}$ In countries like Saudi Arabia, female gender is believed to be a barrier to optimal diabetes management due to limitations of health education. Women also have lower levels of physical activity compared to men due to time-consuming domestic responsibilities as well as a lack of culturally appropriate outdoor facilities. ${ }^{100}$

Traditional Italian cuisine is that of the Mediterranean diet, including controlled quantities of fats, a low percentage of carbohydrates, a low glycemic index and a high content in dietary fiber. ${ }^{101}$ Pasta, traditionally an Italian dish, has expanded to many countries and has become a major dietary source of energy globally. Somewhat counterintuitively, several studies have found the glycemic index of pasta to be low to moderate compared to other starchy foods such as white bread and potatoes. Cross sectional analysis demonstrated that higher pasta intake was associated with better adherence to the Mediterranean diet. ${ }^{102}$ However, over the years there has been a decrease in adherence to the Mediterranean dietary pattern. This is thought to be secondary to several factors, including changes in socio-cultural, intergenerational, and economic factors, such as increased income. Poor adherence is also believed to be a consequence of the evolving female gender role, different organization of working hours, urbanization and globalization. ${ }^{103}$

\section{Religious Barriers}

Similar to cultural traditions, dietary patterns and adherence to specific diets can be influenced by religion. For example, studies have shown a short-term increase in caloric intake with corresponding weight increase and an increase in glycemic and lipid markers due to Christmas festivities. ${ }^{104}$

Several recent publications have shown that the nutritional changes observed during Ramadan are associated with unhealthy dietary changes. During observance, individuals will consume a predawn meal, to obtain requirements of water, carbohydrates and energy needed for daytime hours. This meal varies slightly by region but typically consists of bread/cereal, couscous, eggs, cheese, and/or rice. The sunset meal that breaks the fast can average up to $150 \mathrm{~g}$ of carbohydrates and over $1200 \mathrm{kcal}$ and usually includes dates, meat or cheese pastries and rice. ${ }^{105,106}$ This is traditionally followed by an additional meal eaten a few hours later or nocturnal grazing. ${ }^{106}$ One food survey of 340 Moroccan households found significant increases in energy intake, carbohydrate intake, sucrose intake, sodium intake, and calcium intake and a significant decrease in protein and lipid intake. Carbohydrates increased from $312 \mathrm{~g}$ /day before Ramadan to $360 \mathrm{~g}$ /day during Ramadan. This was possibly due to increased consumption of cereal products, which are popular in this period. An atmosphere of festive solidarity and family grouping is also thought to contribute to the changes in dietary habits. ${ }^{107}$

Yom Kippur (Jewish Day of Atonement) is considered the holiest day of the Jewish calendar and is one of two times of the year that people of Jewish faith abstain from food and water for 25 hours (from sunset on day 1 to one hour after sunset the following day). The other day long fast is Tish B'Av, also known as Ninth of Av, but there are 4 additional periods of potential fasting of shorter duration within the Jewish faith. ${ }^{108}$

The religious group Seventh-day Adventists promotes vegetarianism, which may be advantageous as several studies have shown that vegetarianism is associated with lower prevalence of type 2 diabetes. ${ }^{109}$ However, the diet of Seventhday Adventists is characterized by a large intake of carbohydrates in the form of fruits and vegetables, as well as high content of fatty acids, dietary fiber, folic acid, vitamin $\mathrm{C}$, vitamin $\mathrm{E}$ and $\mathrm{Mg}^{110}$ Vegetarianism is also practiced in Hinduism and Buddhism. ${ }^{111,112}$ Though dietary practices vary, the traditional diets of Hindu families include 
carbohydrates as a staple, usually rice and or chapatis (form of bread). ${ }^{113}$ Lin et al investigated vegetarian practices of Hindus and Buddhist in Malaysia and found that rice was a major dietary staple and was consumed daily by majority of participants. Other carbohydrates consumed included porridge, rice noodles and bread. Buddhism participants consumed higher daily intake of carbs compared to Hindu ones with a carbohydrate intake of $67.7 \%$ of total energy intake and $63.55 \%$, respectively. ${ }^{114}$

\section{Economic Barriers}

People of lower socioeconomic status often face unique challenges in adhering to planned dietary changes. One barrier unique to people with lower economic security is food insecurity - the disruption of food intake or eating patterns due to the cost of food items exceeding an individual's or family's financial circumstances. ${ }^{115}$ Food insecurity is associated with a higher hemoglobin A1c. ${ }^{116}$ Low carbohydrate eating patterns are potentially more expensive than higher carbohydrate plans, although one study regarded the potential increased cost as negligible. ${ }^{117,118}$ This could potentially limit access (or perceived access) to this eating pattern for people with inadequate financial resources.

\section{Patient Preference}

A potential component of adherence that is difficult to quantify is patient buy in. In studies of low carbohydrate dietary interventions, randomized trials such as those by Guldbrand and Iqbal, ${ }^{58,69}$ tend to have poorer rates of adherence than non-randomized trials such as those by Hussein and Hallberg. ${ }^{35,53}$ In a 24-week study by Hussain in which obese participants chose between a VLCKD and a low-calorie diet, participants with diabetes who chose a VLCKD lost $12 \%$ of their body weight, whereas those who chose a low-calorie eating plan lost $7 \% .{ }^{35}$ In a year-long intervention, Hallberg found that patients with type 2 diabetes on a VLCKD experienced a $12 \%$ mean weight loss and $17 \%$ reduction in hemoglobin Alc. Health coaches monitored ketones and adjusted diet as needed to achieve ketosis. Participants received education either on-site or via web-based recorded content and also received remote care from health coaches. The study had a very low attrition rate of $16.8 \%$ from the VLCKD group. ${ }^{53}$ Two-year follow-up showed more modest improvements in glycemic control with $\mathrm{HbA} 1 \mathrm{c}$ reductions of $0.6-0.9 \%$ observed in those on VLCKDs, which may be in part due to the higher attrition rate over longer observation periods. ${ }^{64,119,120}$ This suggests that there may be an element of patient "buy-in" to the dietary strategy that promotes adherence.

\section{Potential Solutions}

Overcoming barriers to dietary adherence in patients with diabetes is critical to reducing disease burden and preventing its progression. Objective self-monitoring of carbohydrate intake can help maintain dietary adherence. Validated examples include the use of home biomarkers such as urinary ketone levels and blood or breath ketone meters. ${ }^{121-123}$ Urbain et al examined serum and urine ketones during the 6th week of a VLCKD. Urine ketones were measured using over-the-counter reagent strips that determined the presence of acetoacetate. The levels of blood and urine ketone bodies correlated throughout the 24-hour period. The best time for urine testing for ketosis was early morning and several hours after dinner. ${ }^{51}$ Hand-held ketone sensors using a fingerstick obtained capillary blood sample accurately measure BHB in starvation-induced ketonemia when compared with the gold standard, venous whole blood by an enzymatic laboratory reference method. ${ }^{124}$ More affordable meters have also shown good correlation. Moore compared the Precision Xtra meter with the more affordable Meter 2- Keto-Mojo in a double-blind cross-over study. Ketone and glucose levels were measured before and twice after ingesting racemic ketone, natural ketone, or maltodextrin supplement. Both meters had excellent agreement between each other for measuring ketone measurement, including the more affordable Meter $2 .{ }^{125}$

In office monitoring of other biochemical markers is also helpful. As described above, significant improvements in blood glucose, HbA1c, HDL cholesterol and triglyceride levels are consistently noted in those who following a VLKD and therefore could be used as markers of adherence. Weight loss and improvements in blood pressure have also been noted but are less specific to an LCD.

Accuracy issues notwithstanding, another potential tool to improve adherence may be through self-monitoring of food intake, eg, using a food diary. Burke's systematic review of self-monitoring in weight management found significant associations between monitoring frequency and weight loss consistently in the 15 studies assessing dietary self- 
monitoring. However, the level of evidence was weak due to methodologic limitations. ${ }^{126}$ Though a food diary has the limitations previously discussed, when used as the sole tool for assessing adherence, it may play a role in improving the mindfulness of the types and amounts of foods consumed, thereby indirectly helping adherence.

Educational interventions may also be a means of improving adherence. In a Swedish study, an educational course on carbohydrate restricted diet (75 g/day or less) and corresponding insulin dose reduction was used to enhance adherence among patients with type 1 diabetes over four years. After 2 years, about $50 \%$ achieved stable lowering of $\mathrm{HbA} 1 \mathrm{c} .{ }^{127} \mathrm{In}$ addition, continuous care models integrating telemedicine are a novel approach that has been effective at guiding nutritional ketosis and assisting with adherence. ${ }^{120}$

\section{Future Research Directions}

Research has identified multiple methods to measure and improve dietary adherence to LCD. Given the bias associated with self-reported dietary intake, studies comparing various objective measures of dietary adherence are needed to determine the optimal metric. More research is also needed to better understand the potential role for weight loss medications as adjunct therapy to assist adherence to an LCD, as well as the myriad of cultural, psychological, and economic factors that play a role in adherence. Additionally, the long-term effects of VLCKD on biochemical parameters requires further investigation to help individualize therapy.

\section{Disclosure}

Dr William S Yancy Jr reports personal fees from dietdoctor.com. The authors report no other conflicts of interest in this work.

\section{References}

1. WHO. Diabetes fact sheet; 2020. Available from: https://www.who.int/news-room/fact-sheets/detail/diabetes. Accessed January $12,2022$.

2. Morgan CL, Currie CJ, Peters JR. Relationship between diabetes and mortality: a population study using record linkage. Diabetes Care. 2000;23(8):1103-1107. doi:10.2337/diacare.23.8.1103

3. Edelstein SL, Knowler WC, Bain RP, et al. Predictors of progression from impaired glucose tolerance to NIDDM: an analysis of six prospective studies. Diabetes. 1997;46(4):701-710. doi:10.2337/diab.46.4.701

4. Knowler WC, Barrett-Connor E, Fowler SE, et al. Reduction in the incidence of type 2 diabetes with lifestyle intervention or metformin. $N$ Engl $J$ Med. 2002;346(6):393-403.

5. Tuomilehto J, Lindstrom J, Eriksson JG, et al. Prevention of type 2 diabetes mellitus by changes in lifestyle among subjects with impaired glucose tolerance. $N$ Engl J Med. 2001;344(18):1343-1350. doi:10.1056/NEJM200105033441801

6. American Diabetes Association. 5. Facilitating behavior change and well-being to improve health outcomes: standards of medical care in diabetes-2020. Diabetes Care. 2020;43(Supp11):S48-S65. doi:10.2337/dc20-S005

7. Evert AB, Dennison M, Gardner CD, et al. Nutrition Therapy for Adults With Diabetes or Prediabetes: a Consensus Report. Diabetes Care. 2019;42(5):731-754. doi:10.2337/dci19-0014

8. Franz MJ, MacLeod J, Evert A, et al. Academy of nutrition and dietetics nutrition practice guideline for type 1 and type 2 diabetes in adults: systematic review of evidence for medical nutrition therapy effectiveness and recommendations for integration into the nutrition care process. $J$ Acad Nutr Diet. 2017;117(10):1659-1679. doi:10.1016/j.jand.2017.03.022

9. MacLeod J, Franz MJ, Handu D, et al. Academy of nutrition and dietetics nutrition practice guideline for type 1 and type 2 diabetes in adults: nutrition intervention evidence reviews and recommendations. $J$ Acad Nutr Diet. 2017;117(10):1637-1658. doi:10.1016/j.jand.2017.03.023

10. Merrill JD, Soliman D, Kumar N, Lim S, Shariff AI, Yancy WS. Low-carbohydrate and very-low-carbohydrate diets in patients with diabetes. Diabetes Spectr. 2020;33(2):133-142. doi:10.2337/ds19-0070

11. Gibson AA, Sainsbury A. Strategies to improve adherence to dietary weight loss interventions in research and real-world settings. Behav Sci (Basel). 2017;7(3). doi:10.3390/bs7030044

12. Dansinger ML, Gleason JA, Griffith JL, Selker HP, Schaefer EJ. Comparison of the Atkins, Ornish, Weight Watchers, and Zone diets for weight loss and heart disease risk reduction: a randomized trial. JAMA. 2005;293(1):43-53. doi:10.1001/jama.293.1.43

13. Cruwys T, Norwood R, Chachay VS, Ntontis E, Sheffield J. "An important part of who i am": the predictors of dietary adherence among weight-loss, vegetarian, vegan, paleo, and gluten-free dietary groups. Nutrients. 2020;12(4):970. doi:10.3390/nu12040970

14. Elfhag K, Rossner S. Who succeeds in maintaining weight loss? A conceptual review of factors associated with weight loss maintenance and weight regain. Obes Rev. 2005;6(1):67-85. doi:10.1111/j.1467-789X.2005.00170.x

15. Canetti L, Berry EM, Elizur Y. Psychosocial predictors of weight loss and psychological adjustment following bariatric surgery and a weightloss program: the mediating role of emotional eating. Int J Eat Disord. 2009;42(2):109-117. doi:10.1002/eat.20592

16. Osler W, McCrae T. The Principles and Practice of Medicine. New York, London: D. Appleton and company; 1920:xxiv, 1168.

17. Westman EC, Yancy WS, Humphreys M. Dietary treatment of diabetes mellitus in the pre-insulin era (1914-1922). Perspect Biol Med. 2006;49 (1):77-83. doi:10.1353/pbm.2006.0017

18. Wheless JW. History of the ketogenic diet. Epilepsia. 2008;49(Suppl 8):3-5. doi:10.1111/j.1528-1167.2008.01821.x

19. Peterman M. The ketogenic diet in epilepsy. JAMA. 1925;84(26):1979-1983. doi:10.1001/jama.1925.02660520007003 
20. Allen F, Stillman E, Fitz R. Total Dietary Regulation in the Treatment of Diabetes. New York: The Rocckefeller Institue for Medical Research; 1919.

21. Lundgren JR, Janus C, Jensen SBK, et al. Healthy weight loss maintenance with exercise, liraglutide, or both combined. $N$ Engl $J$ Med. 2021;384(18):1719-1730. doi:10.1056/NEJMoa2028198

22. Kossoff EH, McGrogan JR, Bluml RM, Pillas DJ, Rubenstein JE, Vining EP. A modified Atkins diet is effective for the treatment of intractable pediatric epilepsy. Epilepsia. 2006;47(2):421-424. doi:10.1111/j.1528-1167.2006.00438.x

23. Ye F, Li XJ, Jiang WL, Sun HB, Liu J. Efficacy of and patient compliance with a ketogenic diet in adults with intractable epilepsy: a metaanalysis. J Clin Neurol. 2015;11(1):26-31. doi:10.3988/jen.2015.11.1.26

24. Kossoff EH, Cervenka MC, Henry BJ, Haney CA, Turner Z. A decade of the modified Atkins diet (2003-2013): results, insights, and future directions. Epilepsy Behav. 2013;29(3):437-442. doi:10.1016/j.yebeh.2013.09.032

25. Sirven J, Whedon B, Caplan D, et al. The ketogenic diet for intractable epilepsy in adults: preliminary results. Epilepsia. 1999;40(12):17211726. doi:10.1111/j.1528-1157.1999.tb01589.x

26. Klein P, Janousek J, Barber A, Weissberger R. Ketogenic diet treatment in adults with refractory epilepsy. Epilepsy Behav. $2010 ; 19(4): 575-579$. doi:10.1016/j.yebeh.2010.09.016

27. Cervenka MC, Henry B, Nathan J, Wood S, Volek JS. Worldwide dietary therapies for adults with epilepsy and other disorders. J Child Neurol. 2013;28(8):1034-1040. doi:10.1177/0883073813488671

28. Nei M, Ngo L, Sirven JI, Sperling MR. Ketogenic diet in adolescents and adults with epilepsy. Seizure. 2014;23(6):439-442. doi:10.1016/j. seizure.2014.02.015

29. Freedman MR, King J, Kennedy E. Popular diets: a scientific review. Obes Res. 2001;9(Suppl 1):1S-40S. doi:10.1038/oby.2001.113

30. Shan Z, Rehm CD, Rogers G, et al. Trends in dietary carbohydrate, protein, and fat intake and diet quality among US adults, 1999-2016. JAMA. 2019;322(12):1178-1187. doi:10.1001/jama.2019.13771

31. Vitolins MZ, Anderson AM, Delahanty L, et al. Action for Health in Diabetes (Look AHEAD) trial: baseline evaluation of selected nutrients and food group intake. J Am Diet Assoc. 2009;109(8):1367-1375. doi:10.1016/j.jada.2009.05.016

32. Oza-Frank R, Cheng YJ, Narayan KM, Gregg EW. Trends in nutrient intake among adults with diabetes in the United States: 1988-2004. J Am Diet Assoc. 2009;109(7):1173-1178. doi:10.1016/j.jada.2009.04.007

33. Sheard NF, Clark NG, Brand-Miller JC, et al. Dietary carbohydrate (amount and type) in the prevention and management of diabetes: a statement by the American diabetes association. Diabetes Care. 2004;27(9):2266-2271. doi:10.2337/diacare.27.9.2266

34. Yancy WS, Foy M, Chalecki AM, Vernon MC, Westman EC. A low-carbohydrate, ketogenic diet to treat type 2 diabetes. Nutr Metab (Lond). 2005;2:34. doi:10.1186/1743-7075-2-34

35. Hussain TA, Mathew TC, Dashti AA, Asfar S, Al-Zaid N, Dashti HM. Effect of low-calorie versus low-carbohydrate ketogenic diet in type 2 diabetes. Nutrition. 2012;28(10):1016-1021. doi:10.1016/j.nut.2012.01.016

36. van Zuuren EJ, Fedorowicz Z, Kuijpers T, Pijl H. Effects of low-carbohydrate- compared with low-fat-diet interventions on metabolic control in people with type 2 diabetes: a systematic review including GRADE assessments. Am J Clin Nutr. 2018;108(2):300-331. doi:10.1093/ajcn/ nqy096

37. Westman EC, Yancy WS, Mavropoulos JC, Marquart M, McDuffie JR. The effect of a low-carbohydrate, ketogenic diet versus a low-glycemic index diet on glycemic control in type 2 diabetes mellitus. Nutr Metab (Lond). 2008;5:36. doi:10.1186/1743-7075-5-36

38. Accurso A, Bernstein RK, Dahlqvist A, et al. Dietary carbohydrate restriction in type 2 diabetes mellitus and metabolic syndrome: time for a critical appraisal. Nutr Metab (Lond). 2008;5:9. doi:10.1186/1743-7075-5-9

39. Agatston A. The South Beach Diet: The Delicious, Doctor-Designed, Foolproof Plan for Fast and Healthy Weight Loss. Emmaus, Penn.: Rodale; Distributed to the book trade by St. Martin's Press; 2003:ix, 310.

40. Sears B, Lawren B. The Zone: A Dietary Road Map. 1st ed. New York, NY: Regan Books; 1995:xvii, 286.

41. Meng Y, Bai H, Wang S, Li Z, Wang Q, Chen L. Efficacy of low carbohydrate diet for type 2 diabetes mellitus management: a systematic review and meta-analysis of randomized controlled trials. Diabetes Res Clin Pract. 2017;131:124-131. doi:10.1016/j.diabres.2017.07.006

42. Goldenberg JZ, Day A, Brinkworth GD, et al. Efficacy and safety of low and very low carbohydrate diets for type 2 diabetes remission: systematic review and meta-analysis of published and unpublished randomized trial data. BMJ. 2021;372:m4743. doi:10.1136/bmj.m4743

43. Kirkpatrick CF, Bolick JP, Kris-Etherton PM, et al. Review of current evidence and clinical recommendations on the effects of lowcarbohydrate and very-low-carbohydrate (including ketogenic) diets for the management of body weight and other cardiometabolic risk factors: a scientific statement from the National Lipid Association Nutrition and Lifestyle Task Force. J Clin Lipidol. 2019;13(5):689-711.e1. doi:10.1016/j.jacl.2019.08.003

44. Fechner E, Smeets ET, Schrauwen P, Mensink RP. The effects of different degrees of carbohydrate restriction and carbohydrate replacement on cardiometabolic risk markers in humans-a systematic review and meta-analysis. Nutrients. 2020;12(4):991. doi:10.3390/nu12040991

45. Sainsbury E, Kizirian NV, Partridge SR, Gill T, Colagiuri S, Gibson AA. Effect of dietary carbohydrate restriction on glycemic control in adults with diabetes: a systematic review and meta-analysis. Diabetes Res Clin Pract. 2018;139:239-252. doi:10.1016/j.diabres.2018.02.026

46. Snorgaard O, Poulsen GM, Andersen HK, Astrup A. Systematic review and meta-analysis of dietary carbohydrate restriction in patients with type 2 diabetes. BMJ Open Diabetes Res Care. 2017;5(1):e000354. doi:10.1136/bmjdrc-2016-000354

47. Paoli A, Rubini A, Volek JS, Grimaldi KA. Beyond weight loss: a review of the therapeutic uses of very-low-carbohydrate (ketogenic) diets Eur J Clin Nutr. 2013;67(8):789-796. doi:10.1038/ejen.2013.116

48. Feinman RD, Pogozelski WK, Astrup A, et al. Dietary carbohydrate restriction as the first approach in diabetes management: critical review and evidence base. Nutrition. 2015;31(1):1-13. doi:10.1016/j.nut.2014.06.011

49. Atkins RC. Dr. Atkins' Diet Revolution; the High Calorie Way to Stay Thin Forever. New York: D. McKay Co.; 1972:x, 310.

50. Atkins RC. Dr. Atkins' New Diet Revolution. 1st Avon pbk. ed. New York: Avon Books; 2002:xiv, 540.

51. Urbain P, Bertz H. Monitoring for compliance with a ketogenic diet: what is the best time of day to test for urinary ketosis? Nutr Metab (Lond) 2016;13:77. doi:10.1186/s12986-016-0136-4

52. McKenzie AL, Hallberg SJ, Creighton BC, et al. A novel intervention including individualized nutritional recommendations reduces hemoglobin a1c level, medication use, and weight in type 2 diabetes. JMIR Diabetes. 2017;2(1):e5. doi:10.2196/diabetes.6981 
53. Hallberg SJ, McKenzie AL, Williams PT, et al. Effectiveness and safety of a novel care model for the management of type 2 diabetes at 1 year: an open-label, non-randomized, controlled study. Diabetes Ther. 2018;9(2):583-612. doi:10.1007/s13300-018-0373-9

54. Davis NJ, Tomuta N, Schechter C, et al. Comparative study of the effects of a 1-year dietary intervention of a low-carbohydrate diet versus a low-fat diet on weight and glycemic control in type 2 diabetes. Diabetes Care. 2009;32(7):1147-1152. doi:10.2337/dc08-2108

55. Yancy WS, Olsen MK, Guyton JR, Bakst RP, Westman EC. A low-carbohydrate, ketogenic diet versus a low-fat diet to treat obesity and hyperlipidemia: a randomized, controlled trial. Ann Intern Med. 2004;140(10):769-777.

56. Saslow LR, Kim S, Daubenmier JJ, et al. A randomized pilot trial of a moderate carbohydrate diet compared to a very low carbohydrate diet in overweight or obese individuals with type 2 diabetes mellitus or prediabetes. PLoS One. 2014;9(4):e91027. doi:10.1371/journal.pone.0091027

57. Guldbrand H, Dizdar B, Bunjaku B, et al. In type 2 diabetes, randomisation to advice to follow a low-carbohydrate diet transiently improves glycaemic control compared with advice to follow a low-fat diet producing a similar weight loss. Diabetologia. 2012;55(8):2118-2127. doi:10.1007/s00125-012-2567-4

58. Guldbrand H, Dizdar B, Bunjaku B, et al. In type 2 diabetes, randomisation to advice to follow a low-carbohydrate diet transiently improves glycaemic control compared with advice to follow a low-fat diet producing a similar weight loss. Diabetologia. 2012;55(8):2118-2127.

59. Choi YJ, Jeon SM, Shin S. Impact of a ketogenic diet on metabolic parameters in patients with obesity or overweight and with or without type 2 diabetes: a meta-analysis of randomized controlled trials. Nutrients. 2020;12(7):2005. doi:10.3390/nu12072005

60. Westman EC, Yancy WS, Olsen MK, Dudley T, Guyton JR. Effect of a low-carbohydrate, ketogenic diet program compared to a low-fat diet on fasting lipoprotein subclasses. Int J Cardiol. 2006;110(2):212-216. doi:10.1016/j.ijcard.2005.08.034

61. Saslow LR, Daubenmier JJ, Moskowitz JT, et al. Twelve-month outcomes of a randomized trial of a moderate-carbohydrate versus very lowcarbohydrate diet in overweight adults with type 2 diabetes mellitus or prediabetes. Nutr Diabetes. 2017;7(12):1-6. doi:10.1038/s41387-0170006-9

62. Westman EC, Yancy WS, Mavropoulos JC, Marquart M, McDuffie JR. The effect of a low-carbohydrate, ketogenic diet versus a low-glycemic index diet on glycemic control in type 2 diabetes mellitus. Nutr Metab. 2008;5(1):1-9.

63. Mayer SB, Jeffreys AS, Olsen MK, McDuffie JR, Feinglos MN, Yancy WS. Two diets with different haemoglobin A1c and antiglycaemic medication effects despite similar weight loss in type 2 diabetes. Diabetes Obes Metab. 2014;16(1):90-93. doi:10.1111/dom.12191

64. Tay J, Thompson CH, Luscombe-Marsh ND, et al. Effects of an energy-restricted low-carbohydrate, high unsaturated fat/low saturated fat diet versus a high-carbohydrate, low-fat diet in type 2 diabetes: a 2-year randomized clinical trial. Diabetes Obes Metab. 2018;20(4):858-871. doi: $10.1111 /$ dom. 13164

65. Buse JB, Caprio S, Cefalu WT, et al. How do we define cure of diabetes? Diabetes Care. 2009;32(11):2133-2135. doi:10.2337/dc09-9036

66. De Geest S, Sabate E. Adherence to long-term therapies: evidence for action. Eur J Cardiovasc Nurs. 2003;2(4):323. doi:10.1016/S1474-5151 (03)00091-4

67. Kipnis V, Midthune D, Freedman L, et al. Bias in dietary-report instruments and its implications for nutritional epidemiology. Public Health Nutr. 2002;5(6A):915-923. doi:10.1079/PHN2002383

68. Subar AF, Freedman LS, Tooze JA, et al. Addressing current criticism regarding the value of self-report dietary data. $J$ Nutr. 2015;145 (12):2639-2645. doi:10.3945/jn.115.219634

69. Iqbal N, Vetter ML, Moore RH, et al. Effects of a low-intensity intervention that prescribed a low-carbohydrate vs. a low-fat diet in obese, diabetic participants. Obesity (Silver Spring). 2010;18(9):1733-1738. doi:10.1038/oby.2009.460

70. Hu T, Yao L, Reynolds K, et al. Adherence to low-carbohydrate and low-fat diets in relation to weight loss and cardiovascular risk factors. Obes Sci Pract. 2016;2(1):24-31. doi:10.1002/osp4.23

71. Tay J, Luscombe-Marsh ND, Thompson CH, et al. Comparison of low- and high-carbohydrate diets for type 2 diabetes management: a randomized trial. Am J Clin Nutr. 2015;102(4):780-790. doi:10.3945/ajen.115.112581

72. Leng G, Adan RAH, Belot M, et al. The determinants of food choice. Proc Nutr Soc. 2017;76(3):316-327. doi:10.1017/S002966511600286X

73. Iwelunmor J, Newsome V, Airhihenbuwa CO. Framing the impact of culture on health: a systematic review of the PEN-3 cultural model and its application in public health research and interventions. Ethn Health. 2014;19(1):20-46. doi:10.1080/13557858.2013.857768

74. Sohal T, Sohal P, King-Shier KM, Khan NA. Barriers and facilitators for type-2 diabetes management in South Asians: a systematic review. PLoS One. 2015;10(9):e0136202. doi:10.1371/journal.pone.0136202

75. van Dam RM, Global A. Perspective on white rice consumption and risk of type 2 diabetes. Diabetes Care. 2020;43(11):2625-2627. doi: $10.2337 / \mathrm{dci} 20-0042$

76. Frost G. GLUCOSE | glucose tolerance and the glycemic (glycaemic) index. In: Caballero B, editor. Encyclopedia of Food Sciences and Nutrition. Second ed. Academic Press; 2003:2916-2922.

77. Zhao J, Sun J, Su C. Gender differences in the relationship between dietary energy and macronutrients intake and body weight outcomes in Chinese adults. Nutr J. 2020;19(1):45. doi:10.1186/s12937-020-00564-6

78. Lee YJ, Song S, Song Y. High-carbohydrate diets and food patterns and their associations with metabolic disease in the Korean population. Yonsei Med J. 2018;59(7):834-842. doi:10.3349/ymj.2018.59.7.834

79. Kim DY, Kim Y, Lim H. Glycaemic indices and glycaemic loads of common Korean carbohydrate-rich foods. Br J Nutr. 2019;121(4):416-425. doi:10.1017/S0007114518003446

80. Sowattanangoon N, Kotchabhakdi N, Petrie KJ. The influence of Thai culture on diabetes perceptions and management. Diabetes Res Clin Pract. 2009;84(3):245-251. doi:10.1016/j.diabres.2009.02.011

81. Cooper NC. Culture Shock: A Survival Guide to Customs and Etiquette. Asia: Marshall Cavendish International; 2005.

82. Mora N, Golden SH. Understanding cultural influences on dietary habits in Asian, Middle Eastern, and Latino patients with type 2 diabetes: a review of current literature and future directions. Curr Diab Rep. 2017;17(12):126. doi:10.1007/s11892-017-0952-6

83. Balagopal P, Kamalamma N, Patel TG, Misra R. A community-based participatory diabetes prevention and management intervention in rural India using community health workers. Diabetes Educ. 2012;38(6):822-834. doi:10.1177/0145721712459890

84. Joshi SR, Bhansali A, Bajaj S, et al. Results from a dietary survey in an Indian T2DM population: a STARCH study. BMJ Open. 2014;4(10): e005138. doi:10.1136/bmjopen-2014-005138

85. Gupta SS, Teede H, Aroni R. Spicing up your advice for South Asian and Anglo-Australians with type 2 diabetes and CVD: do cultural constructions of diet matter? Appetite. 2018;120:679-697. doi:10.1016/j.appet.2017.10.007 
86. Kulkarni KD. Food, culture, and diabetes in the United States. Clin Diabetes. 2004;22(4):190-192. doi:10.2337/diaclin.22.4.190

87. Arias-Gastelum M, Lindberg NM, Leo MC, et al. Dietary patterns with healthy and unhealthy traits among overweight/obese Hispanic women with or at high risk for type 2 diabetes. J Racial Ethn Health Disparities. 2020;8:293-303. doi:10.1007/s40615-020-00782-y

88. Ogden CL, Fryar CD, Flegal KM. Prevalence of obesity among adults and youth: United States, 2011-2014. In: Statistics NCfH. Centers for Disease Control and Prevention; 2015.

89. Sofianou A, Fung TT, Tucker KL. Differences in diet pattern adherence by nativity and duration of US residence in the Mexican-American population. J Am Diet Assoc. 2011;111(10):1563-9 e2. doi:10.1016/j.jada.2011.07.005

90. Spanakis EK, Golden SH. Race/ethnic difference in diabetes and diabetic complications. Curr Diab Rep. 2013;13(6):814-823. doi:10.1007/ s11892-013-0421-9

91. Capodilupo CM, Kim S. Gender and race matter: the importance of considering intersections in Black women's body image. J Couns Psychol. 2014;61(1):37-49. doi:10.1037/a0034597

92. Sumlin LL, Brown SA. Culture and food practices of African American women with type 2 diabetes. Diabetes Educ. 2017;43(6):565-575. doi:10.1177/0145721717730646

93. James DC. Factors influencing food choices, dietary intake, and nutrition-related attitudes among African Americans: application of a culturally sensitive model. Ethn Health. 2004;9(4):349-367. doi:10.1080/1355785042000285375

94. Wong M, Gucciardi E, Li L, Grace SL. Gender and nutrition management in type 2 diabetes. Can J Diet Pract Res. 2005;66(4):215-220. doi:10.3148/66.4.2005.215

95. Willig AL, Richardson BS, Agne A, Cherrington A. Intuitive eating practices among African-American women living with type 2 diabetes: a qualitative study. J Acad Nutr Diet. 2014;114(6):889-896. doi:10.1016/j.jand.2014.02.004

96. Golstein T, Kark JD, Berry EM, Adler B, Ziv E, Raz I. The effect of a low carbohydrate energy-unrestricted diet on weight loss in obese type 2 diabetes patients - A randomized controlled trial. Clin Nutr ESPEN. 2011;6(4):E178-E186.

97. Fahed AC, El-Hage-Sleiman AK, Farhat TI, Nemer GM. Diet, genetics, and disease: a focus on the Middle East and north Africa region. J Nutr Metab. 2012;2012:109037. doi:10.1155/2012/109037

98. Azizi F, Hadaegh F, Hosseinpanah F, et al. Metabolic health in the Middle East and north Africa. Lancet Diabetes Endocrinol. 2019;7(11):866879. doi:10.1016/S2213-8587(19)30179-2

99. Afshin A, Micha R, Khatibzadeh S, et al. The impact of dietary habits and metabolic risk factors on cardiovascular and diabetes mortality in countries of the Middle East and North Africa in 2010: a comparative risk assessment analysis. BMJ Open. 2015;5(5):e06385. doi:10.1136/ bmjopen-2014-006385

100. Almaatouq MA, Al-Arouj M, Amod A, et al. Barriers to the delivery of optimal antidiabetic therapy in the Middle East and Africa. Int $J$ Clin Pract. 2014;68(4):503-511. doi:10.1111/ijcp.12342

101. La Fauci V, Alessi V, Assefa DZ, et al. Mediterranean diet: knowledge and adherence in Italian young people. Clin Ter. 2020;171(5):e437-e43. doi:10.7417/CT.2020.2254

102. Huang M, Li J, Ha MA, Riccardi G, Liu S. A systematic review on the relations between pasta consumption and cardio-metabolic risk factors. Nutr Metab Cardiovasc Dis. 2017;27(11):939-948. doi:10.1016/j.numecd.2017.07.005

103. Francesco Bottalico XM, Capone R, Bilali HE, Debs P. Erosion of the Mediterranean diet in Apulia Region, South-eastern Italy: exploring socio-cultural and economic dynamics. J Food Nutr Res. 2016;4:258-266.

104. Rees SG, Holman RR, Turner RC. The Christmas feast. Br Med J (Clin Res Ed). 1985;291(6511):1764-1765. doi:10.1136/bmj.291.6511.1764

105. Osman F, Haldar S, Henry CJ. Effects of time-restricted feeding during ramadan on dietary intake, body composition and metabolic outcomes. Nutrients. 2020;12(8):2478. doi:10.3390/nu12082478

106. Monnier L, El Azrak A, Lessan N, Rochd D, Colette C, Bonnet F. Ramadan and diabetes: what we see, learn and understand from continuous glucose monitoring. Diabetes Metab. 2015;41(6):456-462. doi:10.1016/j.diabet.2015.09.002

107. Barakat I, Chamlal H, El Jamal S, Elayachi M, Belahsen R. Food expenditure and food consumption before and during Ramadan in Moroccan households. J Nutr Metab. 2020;2020:8849832. doi:10.1155/2020/8849832

108. Grajower MM. Management of diabetes mellitus on Yom Kippur and other Jewish fast days. Endocr Pract. 2008;14(3):305-311. doi:10.4158/ EP.ep.14.3.305

109. Tonstad S, Stewart K, Oda K, Batech M, Herring RP, Fraser GE. Vegetarian diets and incidence of diabetes in the Adventist Health Study-2. Nutr Metab Cardiovasc Dis. 2013;23(4):292-299. doi:10.1016/j.numecd.2011.07.004

110. Kwok CS, Umar S, Myint PK, Mamas MA, Loke YK. Vegetarian diet, Seventh Day Adventists and risk of cardiovascular mortality: a systematic review and meta-analysis. Int J Cardiol. 2014;176(3):680-686. doi:10.1016/j.ijcard.2014.07.080

111. Chiu THT, Pan WH, Lin MN, Lin CL. Vegetarian diet, change in dietary patterns, and diabetes risk: a prospective study. Nutr Diabetes. 2018;8 (1):12. doi:10.1038/s41387-018-0022-4

112. Leitzmann C. Vegetarian nutrition: past, present, future. Am J Clin Nutr. 2014;100(Suppl 1):496S-502S. doi:10.3945/ajcn.113.071365

113. Nesbitt E. A focus on: Hinduism: the journal of the health visitors' association. Commun Practition. $2018 ; 91(8): 25-27$.

114. Lian CW, Hock CK, Fong TM, Yuvaraj L, Aldrin RD, Llah NIA. Vegetarian practices, body composition and dietary intake among Hindus and Buddhists in Kuching, Sarawak, Malaysia. South East Asia J Public Health. 2019;8(1):3-9. doi:10.3329/seajph.v8i1.42266

115. Patel MR. Social determinants of poor management of type 2 diabetes among the insured. Curr Diab Rep. 2020;20(11):67. doi:10.1007/s11892020-01354-4

116. Berkowitz SA, Karter AJ, Corbie-Smith G, et al. Food insecurity, food "deserts," and glycemic control in patients with diabetes: a longitudinal analysis. Diabetes Care. 2018;41(6):1188-1195. doi:10.2337/dc17-1981

117. Raffensperger JF. The least-cost low-carbohydrate diet is expensive. Nutr Res. 2008;28(1):6-12. doi:10.1016/j.nutres.2007.10.002

118. Zinn C, North S, Donovan K, Muir C, Henderson G. Low-carbohydrate, healthy-fat eating: a cost comparison with national dietary guidelines. Nutr Diet. 2020;77(2):283-291. doi:10.1111/1747-0080.12534

119. Shai I, Schwarzfuchs D, Henkin Y, et al. Weight loss with a low-carbohydrate, Mediterranean, or low-fat diet. $N$ Engl J Med. 2008;359(3):229241. doi:10.1056/NEJMoa0708681 
120. Athinarayanan SJ, Adams RN, Hallberg SJ, et al. Long-term effects of a novel continuous remote care intervention including nutritional ketosis for the management of type 2 diabetes: a 2-year non-randomized clinical trial. Front Endocrinol (Lausanne). 2019;10:348. doi:10.3389/ fendo.2019.00348

121. Guntner AT, Kompalla JF, Landis H, et al. Guiding ketogenic diet with breath acetone sensors. Sensors (Basel). 2018;18(11):3655. doi:10.3390/ s18113655

122. Suntrup Iii DJ, Ratto TV, Ratto M, McCarter JP. Characterization of a high-resolution breath acetone meter for ketosis monitoring. PeerJ. 2020;8:e9969. doi:10.7717/peerj.9969

123. Alkedeh O, Priefer R. The ketogenic diet: breath acetone sensing technology. Biosensors (Basel). 2021;11(1). doi:10.3390/bios11010026

124. Byrne HA, Tieszen KL, Hollis S, Dornan TL, New JP. Evaluation of an electrochemical sensor for measuring blood ketones. Diabetes Care. 2000;23(4):500-503. doi:10.2337/diacare.23.4.500

125. Moore AR, Holland-Winkler AM, Ansley JK, Boone EDH, Schulte MKO. Reliability and diagnostic performance of a new blood ketone and glucose meter in humans. J Int Soc Sports Nutr. 2021;18(1):6. doi:10.1186/s12970-020-00404-2

126. Burke LE, Wang J, Sevick MA. Self-monitoring in weight loss: a systematic review of the literature. J Am Diet Assoc. 2011;111(1):92-102. doi:10.1016/j.jada.2010.10.008

127. Nielsen JV, Gando C, Joensson E, Paulsson C. Low carbohydrate diet in type 1 diabetes, long-term improvement and adherence: a clinical audit. Diabetol Metab Syndr. 2012;4(1):23. doi:10.1186/1758-5996-4-23

\section{Publish your work in this journal}

Diabetes, Metabolic Syndrome and Obesity: Targets and Therapy is an international, peer-reviewed open-access journal committed to the rapid publication of the latest laboratory and clinical findings in the fields of diabetes, metabolic syndrome and obesity research. Original research, review, case reports, hypothesis formation, expert opinion and commentaries are all considered for publication. The manuscript management system is completely online and includes a very quick and fair peer-review system, which is all easy to use. Visit http://www.dovepress. com/testimonials.php to read real quotes from published authors.

Submit your manuscript here: https://www.dovepress.com/diabetes-metabolic-syndrome-and-obesity-targets-and-therapy-journal 